\title{
Método de Faddeev-Jackiw na mecânica clássica
}

\author{
Faddeev-Jackiw method in classical mechanics
}

\author{
L. G. Caro ${ }^{* 1 @, ~ B . ~ M . ~ P i m e n t e l ~}{ }^{1}$, G. E. R. Zambrano ${ }^{2}$ \\ ${ }^{1}$ Universidade Estadual Paulista, Instituto de Física Teórica, 01140-070, São Paulo, SP, Brasil. \\ ${ }^{2}$ Universidad de Nariño, Departamento de Física, San Juan de Pasto, Colombia.
}

Recebido em 30 de julho de 2021. Revisado em 13 de outubro de 2021. Aceito em 20 de outubro de 2021.

\begin{abstract}
As teorias de vínculos são de grande interesse na física teórica, pois todas as teorias conhecidas das interações fundamentais apresentam liberdade de calibre, que é um tipo de caráter singular da teoria, o que implica que ela possui vínculos. Por outro lado, uma vez que a teoria quântica é comumente construída sobre uma estrutura hamiltoniana, o processo de quantização canônica é o mais apropriado se se dispuser da formulação hamiltoniana da teoria clássica. Assim, surge a necessidade de se obter os parênteses de Poisson (PP) das variáveis canônicas na teoria clássica a ser quantizada. O presente trabalho estuda a proposta de Faddeev e Jackiw, revisada por Barcelos-Neto e Wotzasek, para obter os PP em teorias singulares por meio de uma abordagem que faz uso dos elementos geométricos próprios da teoria hamiltoniana. O processo foi construído especificamente na mecânica dos pontos materiais e posteriormente foi implementado em uma série de exemplos, obtendo os PP fundamentais em cada um deles e deixando assim o terreno pronto para a quantização.
\end{abstract}

Palabras-chave: Mecânica clássica, forma simplética, sistemas singulares.

Constrained theories are of great interest in theoretical physics, since all known theories of fundamental interactions present gauge freedom, which is a type of singular feature of the theory and implies that the theory is constrained. On the other hand, since the quantum theory is commonly constructed on a Hamiltonian structure, the canonical quantization process is the most suitable if the Hamiltonian formulation of the classical theory is available. Thus, the necessity to obtain the Poisson Brackets (PB) of the canonical variables in the theory to be quantized arises. In the present work we study the Faddeev and Jackiw proposal, reviewed by Barcelos-Neto and Wotzasek, to obtain the PB in singular theories by means of an approach employing the geometric elements of the Hamiltonian theory themselves. The process was built specifically for matter point mechanics and subsequently was implemented in a sequence of examples, obtaining the fundamental PB in each of them, thus preparing the ground for quantization.

Keywords: Classical mechanics, symplectic form, constrained systems.

\section{Introdução}

Na prática, uma teoria quântica é construída a partir de uma teoria clássica por meio de um processo conhecido como quantização. De fato, a mecânica quântica, tal como a conhecemos, é desenvolvida sobre a estrutura canônica fornecida pela formulação hamiltoniana da mecânica clássica. Porém, após realizada a quantização, muitas vezes a teoria quântica obtida apresenta características que não têm contraparte na abordagem clássica; um claro exemplo é a chamada degenerescência de troca, que é uma consequência da indistinguibilidade das partículas idênticas em mecânica quântica e que foi a motivação para introduzir o princípio de simetrização dos estados correspondentes a sistemas de partículas idênticas. Por outro lado, a necessidade de descrever situações nas quais o número de partículas pode variar conforme o sistema evolui fez com que o conceito de campo visse a luz pela primeira vez na teoria quântica. Submetido às leis da relatividade especial, esse mesmo

\footnotetext{
*Endereço de correspondência: gabriel.caro@unesp.br
}

conceito posteriormente também teve lugar no mundo clássico, nascendo assim a chamada teoria clássica de campos. Foi assim que a arquetípica ideia de partícula pôde ser estendida a o que hoje é conhecido na literatura como campo de matéria. Contudo, os campos já estavam presentes na Física desde que as interações gravitacional e eletromagnética foram descritas com grande sucesso por meio da noção de interação à distância, que matematicamente deu lugar à ideia de campo.

No estudo das interações elementares foi descoberta a invariância da teoria sob um tipo especial de transformações dos campos, a saber, as chamadas transformações de calibre, de onde nasceu o conceito de uma nova forma de simetria: a simetria de calibre. $O$ fato de que uma teoria esteja dotada dessa propriedade realmente é uma evidência de que a nossa descrição não é capaz de fornecer uma dinâmica univocamente determinada, mas de uma série de possibilidades fisicamente equivalentes. De fato, todas as teorias conhecidas das interações fundamentais apresentam essa característica.

A teoria de campos clássicos pode ser vista como uma extensão da mecânica clássica para um número 
infinito de graus de liberdade, o que permite estudá-la por meio das já conhecidas formulações lagrangiana e hamiltoniana e, é claro, as teorias de interação também podem ser tratadas utilizando ditas abordagens. É assim que encontramos que as teorias com liberdade de calibre resultam pertencer ao conjunto das chamadas teorias singulares, que são caracterizadas pelo fato de que a formulação hamiltoniana não pode ser construída, partindo da abordagem lagrangiana, seguindo o algoritmo padrão aprendido em mecânica clássica.

O procedimento de quantização comumente utilizado é conhecido como quantização canônica e foi proposto por Dirac no ano 1925 [1], tendo como principal exigência a existência de uma formulação hamiltoniana bem definida da teoria clássica a ser quantizada. Assim, o fato de não poder, em primeira instância, dispor de uma teoria hamiltoniana de sistemas singulares já é um impedimento para a quantização. Os trabalhos de Bergmann [2] em colaboração com Brunings [3], Penfield, Schiller, Zatzkis [4] e Anderson [5] entre os anos 1949 e 1950 permitiram entender a conexão entre os vínculos presentes em uma teoria singular e as respectivas propriedades de invariância; esses trabalhos foram desenvolvidos no contexto de teorias de campos. Entretanto, Dirac mostrou no ano 1950 que o tratamento de sistemas com vínculos pode ser estudado em sistemas com finitos graus de liberdade (ou sistemas discretos) 6]. O método desenvolvido por Dirac foi realizado no marco de uma teoria puramente hamiltoniana, sendo o objetivo central a disponibilização da quantização canônica. Um dos grandes aportes do método de Dirac foi a classificação dos vínculos em primeira e segunda classe e, mais ainda, ele demostrou que os vínculos de primeira classe resultam ser geradores (no sentido clássico) de transformações de equivalência que no caso contínuo seriam as transformações de calibre. Sem sombra de dúvida, a maior contribuição do método de Dirac foi a introdução dos hoje chamados parênteses de Dirac, que são a versão "corrigida" dos parênteses de Poisson da formulação hamiltoniana padrão e que permitem a correta passagem à teoria quântica. Dirac também estendeu o seu método à teoria de campos 7 .

Embora a análise dos sistemas com vínculos desenvolvida por Dirac e Bergmann tenha tido um inegável e grande sucesso, vários anos mais tarde Faddeev e Jackiw (F-J) apresentaram uma proposta, bastante geral, para o tratamento de sistemas singulares [8] sem ter que recorrer à classificação dos vínculos em primeira ou segunda classe que foi estabelecida no método de Dirac. O método F-J, chamado também por alguns autores de método simplético, pode ser visto como um caminho geométrico para a obtenção dos parênteses a serem quantizados. Além do fato de evadir a classificação dos vínculos, uma vantagem do método F-J é que ele fornece os verdadeiros vínculos da teoria e estes resultam ser perfeitamente consistentes com as equações de movimento, o que permite distinguir os verdadeiros graus de liberdade físicos do sistema. Poucos anos depois, Barcelos-Neto e Wotzasek (BN-W) propuseram uma alternativa (um pouco mais algébrica) ao método F-J [9, 10] que permite incorporar os próprios vínculos de maneira iterativa no caminho a obter a estrutura simplética que nos permitirá determinar diretamente os verdadeiros parênteses das variáveis canônicas correspondentes aos graus de liberdade dinâmicos.

O presente trabalho é estruturado como segue: $\mathrm{Na}$ seção 2 apresentamos, de maneira sequenciada, as ideias centrais da mecânica à la Faddeev-Jackiw, inicialmente para sistemas regulares. Posteriormente, citamos a proposta original de Faddeev e Jackiw para enfrentar o problema dos sistemas singulares e também mencionamos a dificuldade de sua implementação. Na seção 3 contemplamos, com grande detalhe, a contribuição de Barcelos-Neto e Wotsazek que é especificamente para sistemas singulares. Cabe mencionar que temos escolhido um exemplo específico para acompanhar cada etapa ao longo do desenvolvimento das seções já referidas. Na seção 4 apresentamos uma série de exemplos de sistemas singulares nos quais aplicamos as técnicas estudadas nas seções prévias. Finalmente, na seção 5 destacamos as principais vantagens do método F-J e discutimos os resultados obtidos.

\section{Formulação da mecânica $\grave{a} l a$ Faddeev-Jackiw}

O ponto de partida da formulação de Faddeev-Jackiw é reescrever a lagrangiana do sistema de maneira tal que seja linear nas velocidades $\mathrm{I}^{1}$ isto é, que tenha a forma:

$$
L(\xi ; \dot{\xi})=\theta_{\nu}(\xi) \dot{\xi}^{\nu}-H(\xi),
$$

em que os $\left\{\xi^{\nu}\right\}_{\nu=1}^{N}$ sejam as coordenadas do conjunto que chamaremos de espaço de fase $(M)$ e $H$, a hamiltoniana correspondente; os coeficientes das velocidades são as componentes (na base padrã ${ }^{2}$ ) de um objeto chamado de 1-forma canônica:

$$
\theta=\theta_{1} d \xi^{1}+\cdots+\theta_{N} d \xi^{N}=\theta_{\nu} d \xi^{\nu} .
$$

A lagrangiana apresentada em (1) diz-se que está na forma canônica. Para fins didáticos, resulta conveniente escrever (1) da seguinte maneira:

$$
L=\underbrace{\left(\begin{array}{lll}
\theta_{1} & \cdots & \theta_{N}
\end{array}\right)}_{\Theta}\left(\begin{array}{c}
\dot{\xi}^{1} \\
\vdots \\
\dot{\xi}^{N}
\end{array}\right)-H,
$$

em que as componentes da 1 -forma canônica $\theta$ foram organizadas em um vetor linha que temos denotado por

\footnotetext{
${ }^{1} \mathrm{Na}$ presente abordagem chamamos de velocidades as derivadas temporais das coordenadas (que não necessariamente representam os pontos do espaço de configuração).

2 Escolhidas as coordenadas $\xi^{\mu}$, o conjunto $\left\{d \xi^{\mu}\right\}_{\mu=1}^{N}$ é uma base para o espaço de 1 -formas em $M$.
} 
$\Theta$. Nesse sentido $\Theta$ vem a ser apenas uma representação da 1 -forma $\theta$.

É importante mencionar que qualquer lagrangiana pode ser escrita na forma canônica, mas para isso se deve fazer uma escolha apropriada das coordenadas independentes do espaço de fase.

Se, por exemplo, considerarmos as típicas coordenadas $q^{a}$ e $p_{a}$ da mecânica hamiltoniana, elas resultam ser independentes nos sistemas regulares ${ }^{3}$ e, consequentemente, a lagrangiana canônica resulta ser simplesmente:

$$
L=p_{a} \dot{q}^{a}-H \quad(a=1, \cdots, N / 2 \stackrel{!}{=} n),
$$

pois escrita dessa forma, ela já é linear nas velocidades. Assim, bastará fazer com que os $\xi^{\mu}$ desempenhem o papel de coordenadas unificadas; i. e.:

$$
\xi=\left(\begin{array}{c}
q \\
p
\end{array}\right) ; \quad q, p \in \mathbb{R}^{n} .
$$

Com isso, podemos escrever a lagrangiana canônica como:

$$
L=\left(\begin{array}{ll}
p & 0
\end{array}\right)\left(\begin{array}{c}
\dot{q} \\
\dot{p}
\end{array}\right)-H .
$$

Logo, podemos identificar:

$$
\Theta=\left(\begin{array}{ll}
p & 0
\end{array}\right) \stackrel{!}{=}\left(\begin{array}{llllll}
p_{1} & \cdots & p_{n} & 0 & \cdots & 0
\end{array}\right),
$$

e assim, a 1-forma canônica de um sistema regular resulta:

$$
\begin{aligned}
\theta & =p_{1} d q^{1}+\cdots+p_{n} d q^{n}+0 d p_{1}+\cdots 0 d p_{n} \\
& =p_{1} d q^{1}+\cdots+p_{n} d q^{n}=p_{a} d q^{a} .
\end{aligned}
$$

No entanto, quando estamos perante um sistema singular, nem todas as coordenadas resultam ser independentes e isso faz com que devamos escolher cuidadosamente as coordenadas $\xi^{\mu}$. Para ilustrar essa situação, considerese a seguinte lagrangiand 11 :

$$
L=\frac{1}{2} \dot{q}_{1}^{2}+q_{2} \dot{q}_{1}+(1-\alpha) q_{1} \dot{q}_{2}+\frac{\beta}{2}\left(q_{1}-q_{2}\right)^{2},
$$

em que $\alpha$ e $\beta$ são constantes; com $0 \neq \alpha^{2} \neq \beta$, a fim de obtermos uma dinâmica bem definida. Seguindo o procedimento padrão da mecânica hamiltoniana teremos:

$$
\begin{gathered}
p_{1}=\dot{q}_{1}+q_{2}, \quad p_{2}=(1-\alpha) q_{1}, \\
H=\frac{1}{2}\left(p_{1}-q_{2}\right)^{2}-\frac{\beta}{2}\left(q_{1}-q_{2}\right)^{2} .
\end{gathered}
$$

Em primeiro lugar, devemos observar que as coordenadas $p_{2}$ e $q_{1}$ não são independentes; logo, uma boa escolha de coordenadas do espaço de fase será a seguinte:

$$
\xi^{t}=\left(\begin{array}{lll}
q_{1} & q_{2} & p_{1}
\end{array}\right)
$$

\footnotetext{
$3 \mathrm{Na}$ formulação hamiltoniana um sistema é dito regular quando é possível expressar cada uma das velocidades em termos das coordenadas e dos momentos.

4 Vamos trabalhar com este exemplo na medida em que formos construindo e desenvolvendo as etapas do método F-J.
}

Com isso já é simples escrever a lagrangiana (2) na forma canônica:

$$
L=p_{1} \dot{q}_{1}+p_{2} \dot{q}_{2}-H=p_{1} \dot{q}_{1}+(1-\alpha) q_{1} \dot{q}_{2}-H .
$$

Neste caso:

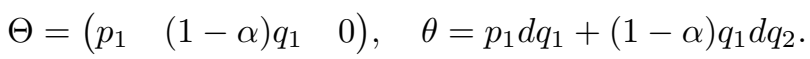

Havíamos mencionado que $\Theta$ é uma representação (matricial) de um objeto chamado de 1-forma canônica; o seguinte será explicar o que é realmente esse objeto e qual é a sua importância na presente abordagem.

No caso geral (regular ou singular) o espaço de fase está equipado com uma 2-forma $\omega$ que contém toda a estrutura canônica do sistema. Encontramos uma evidência disso no fato de que $\omega$ aparece naturalmente nas equações de movimento; por outro lado, o caráter regular ou singular do sistema pode ser determinado apenas conhecendd ${ }^{5} \omega$. A relação entre a 2 -forma $\omega$ e a 1-forma $\theta$ é a seguinte:

$$
\omega \doteq d \theta
$$

em que $d$ é a operação de derivação exterior de formas diferenciais. Como já se viu, a 1 -forma canônica $\theta$ pode ser expressa como:

$$
\theta=\theta_{\nu} d \xi^{\nu} \quad \Rightarrow \quad \Theta=\left(\begin{array}{lll}
\theta_{1} & \cdots & \theta_{N}
\end{array}\right) .
$$

Logo, de (4):

$$
\begin{aligned}
\omega & =d\left(\theta_{\nu} d \xi^{\nu}\right)=\frac{\partial \theta_{\nu}}{\partial \xi^{\mu}} d \xi^{\mu} \wedge d \xi^{\nu} \\
& =\frac{1}{2}\left(\frac{\partial \theta_{\nu}}{\partial \xi^{\mu}}-\frac{\partial \theta_{\mu}}{\partial \xi^{\nu}}\right) d \xi^{\mu} \wedge d \xi^{\nu} .
\end{aligned}
$$

Levando em conta que, em geral, uma 2 - forma $\alpha$ pode ser escrita como:

$$
\alpha=\sum_{i<j} \alpha_{i j} d x^{i} \wedge d x^{j}=\frac{1}{2} \alpha_{i j} d x^{i} \wedge d x^{j},
$$

identificamos as componentes de $\omega$ :

$$
\omega_{\mu \nu}=\frac{\partial \theta_{\nu}}{\partial \xi^{\mu}}-\frac{\partial \theta_{\mu}}{\partial \xi^{\nu}} .
$$

Evidentemente $\omega_{\mu \nu}$ é antissimétrico nos seus índices e, por conseguinte, podemos representar $\omega$ por meio de uma matriz antissimétrica $\Omega$ cujos elementos sejam as componentes $\omega_{\mu \nu}$

$$
\Omega=\left(\begin{array}{cccc}
0 & \omega_{12} & \cdots & \omega_{1 N} \\
-\omega_{12} & 0 & \cdots & \omega_{2 N} \\
\vdots & \vdots & \ddots & \vdots \\
-\omega_{1 N} & -\omega_{2 N} & \cdots & 0
\end{array}\right)
$$

\footnotetext{
$\overline{5}$ De maneira mais precisa, é a representação matricial de $\omega$ que fornece essa informação, como será apresentado mais diante.
} 
Assim, para construir a matriz $\Omega$ bastará identificar os elementos da parte triangular superior (ou inferior); lembrando que a cada elemento da 2 -forma $\omega$ correspondelhe uma entrada na matriz cuja posição é determinada segundd ${ }^{6}$,

$$
\omega=\cdots+\omega_{\mu \nu} \underbrace{d \xi^{\mu}}_{\begin{array}{c}
\mu-\text { éssima } \\
\text { linha }
\end{array}} \wedge \underbrace{d \xi^{\nu}}_{\begin{array}{c}
\nu-\text { éssima } \\
\text { coluna }
\end{array}}+\cdots
$$

Considerando novamente o caso da mecânica hamiltoniana, podemos determinar a 2 -forma $\omega$ de um sistema regular de $n$ graus de liberdade tomando a derivada exterior da 1-forma canônica apresentada em (2). Para esse propósito resulta conveniente mencionar as seguintes propriedades da derivada exterior:

1. Para uma $p$-forma $\alpha$ e uma $q$-forma $\beta$ :

$$
d(\alpha \wedge \beta)=d \alpha \wedge \beta+(-1)^{p} \alpha \wedge d \beta .
$$

2. Para uma $p$-forma qualquer:

$$
d(d \alpha)=0 .
$$

Levando em conta que uma função escalar $f$ é considerada uma 0 -forma, temos o seguinte caso particular da primeira propriedade:

$$
d(f \alpha)=d f \wedge \alpha+f d \alpha \quad(\alpha: p \text {-forma }) .
$$

Com isso, a 2-forma $\omega=d \theta$ será:

$$
\begin{aligned}
\omega= & d\left(p_{1} d q^{1}+\cdots+p_{n} d q^{n}\right) \\
= & d\left(p_{1} d q^{1}\right)+\cdots+d\left(p_{n} d q^{n}\right) \\
= & d p_{1} \wedge d q_{1}+p_{1} d\left(d q^{1}\right)+\cdots \\
& \quad+d p_{n} \wedge d q_{n}+p_{n} d\left(d q^{n}\right) \\
= & d p_{1} \wedge d q_{1}+\cdots+d p_{n} \wedge d q_{n},
\end{aligned}
$$

ou de maneira mais compacta:

$$
\omega=d\left(p_{a} d q^{a}\right)=d p_{a} \wedge d q^{a} .
$$

Logo, seguindo a regra apresentada em (6), podemos construir facilmente a representação matricial:

$$
\Omega=\left(\begin{array}{cc}
0 & -I_{n} \\
I_{n} & 0
\end{array}\right)
$$

em que $I_{n}$ é a matriz identidade em $\mathbb{R}^{n \times n}$.

Agora voltemos ao exemplo da lagrangiana apresentada em (3), para esse caso a 2 -forma $\omega$ resulta:

$$
\begin{aligned}
\omega & =d\left(p_{1} d q_{1}+(1-\alpha) q_{1} d q_{2}\right) \\
& =d p_{1} \wedge d q_{1}+(1-\alpha) d q_{1} \wedge d q_{2},
\end{aligned}
$$

\footnotetext{
$\left.\overline{{ }^{6} \mathrm{O} \text { conjunto }\left\{d \xi^{\mu} \wedge d \xi^{\nu}\right.}\right\}_{\mu<\nu}$ constitui uma base do espaço de 2-formas no espaço de fase $M$.
}

ou matricialmente:

$$
\Omega=\left(\begin{array}{ccc}
0 & 1-\alpha & -1 \\
\alpha-1 & 0 & 0 \\
1 & 0 & 0
\end{array}\right) .
$$

Ora, não devemos perder de vista que sempre que dispormos de uma lagrangiana podemos construir o funcional ação $S[\xi] \doteq \int_{t_{1}}^{t_{2}} L(\xi ; \dot{\xi}) d t$ e obter as equações de movimento em virtude do princípio de Hamilton. Lembre-se que em nosso espaço de fase as coordenadas $\xi^{\mu}$ (e em consequência as variações delas) são independentes; com isto, as equações buscadas resultam:

$$
\frac{\delta S}{\delta \xi^{\mu}}=\left(\frac{\partial \theta_{\nu}}{\partial \xi^{\mu}}-\frac{\partial \theta_{\mu}}{\partial \xi^{\nu}}\right) \dot{\xi}^{\nu}-\frac{\partial H}{\partial \xi^{\mu}}=0,
$$

ou, levando em conta (5):

$$
\omega_{\mu \nu} \dot{\xi}^{\nu}=\frac{\partial H}{\partial \xi^{\mu}},
$$

donde vemos que, como foi antecipado, as componentes da 2 -forma $\omega$ aparecem naturalmente nas equações de movimento nas coordenadas escolhidas para o espaço de fase.

Vale salientar que uma vez que $\omega$ é definida como a derivada exterior de outra forma diferencial (equação (4)) ter-se-á que $d \omega=0$, nesse caso, diz-se que $\omega$ é uma forma fechada. Por outro lado, devemos lembrar que uma forma bilinear é dita não-degenerada se e somente se qualquer representação matricial resulte ser invertível; nesse sentido, essa mesma definição de nãodegenerescência estende-se para qualquer 2-forma (em dimensão finita). Assim, para o caso de $\omega$, diremos que ela é não-degenerada se a matriz $\Omega$ resulta invertível. Pode-se provar também que a não-degenerescência da forma $\omega$ é condição necessária e suficiente para garantir o caráter regular do sistema em estudo.

$\mathrm{Na}$ linguagem de formas, uma 2-forma fechada e nãodegenerada é chamada de simplética enquanto que uma 2-forma fechada e degenerada é dita pré-simplética. Então, com essas definições podemos afirmar que um sistema é regular (singular) quando a 2 -forma $\omega$, definida no espaço de fase, é simplética (pré-simplética).

\subsection{Parênteses de Poisson}

Quando o espaço de fase $M$ é equipado com uma forma simplética $\omega$ (sistemas regulares) é possível definir uma operação bilinear $\{\cdot ; \cdot\}$ sobre o espaço de funções suaves em $M$ (usualmente denotado por $\mathcal{C}^{\infty}(M)$ ) conhecida como parênteses de Poisson (PP). Lembre-se de que, em geral, as trajetórias no espaço de fase são obtidas dando solução às equações de movimento; assim, as coordenadas $\xi^{\mu}$ dos pontos sobre essas curvas tornamse funções do tempo. Nesse sentido, as funções de $\mathcal{C}^{\infty}(M)$ são conhecidas como funções dinâmicas, pois elas evoluem com o tempo; então, se $F=F(\xi(t))$ é 
uma função dinâmica ter-se-á:

$$
\dot{F}=\frac{\partial F}{\partial \xi^{\mu}} \dot{\xi}^{\mu} .
$$

Sob a suposição d€7 que a matriz $\Omega$ é invertível, podemos denotar as componentes da inversa dela por $\omega^{\mu \nu}$ e assim:

$$
\Omega^{-1} \Omega=I \quad \Rightarrow \quad \omega^{\mu \rho} \omega_{\rho \nu}=\delta_{\nu}^{\mu}
$$

Usando esse último resultado na equação de movimento 10) consegue-se:

$$
\dot{\xi}^{\mu}=\omega^{\mu \nu} \frac{\partial H}{\partial \xi^{\nu}} .
$$

Assim, substituindo em 111 resulta:

$$
\dot{F}=\frac{\partial F}{\partial \xi^{\mu}} \omega^{\mu \nu} \frac{\partial H}{\partial \xi^{\nu}} .
$$

Podemos ver em (12) que a hamiltoniana $H$ desempenha um papel fundamental, pois a evolução temporal de qualquer função dinâmica está relacionada com o gradiente de $H$. As transformações no espaço de fase que deixam invariante a estrutura hamiltoniana são chamadas de transformações canônica $8^{8}$ e a própria evolução temporal pode ser vista como uma sequência (contínua) de transformações canônicas infinitesimais que são geradas pela hamiltoniana; esse último fato é o que nos permite introduzir os PP por meio da seguinte exigêncie 9 ?

$$
\dot{F} \doteq\{F ; H\}
$$

É a partir de 13 e 12 que introduzimos a definição dos PP de duas funções dinâmicas $F$ e $G$ quaisquer:

$$
\{F ; G\} \doteq \frac{\partial F}{\partial \xi^{\mu}} \omega^{\mu \nu} \frac{\partial G}{\partial \xi^{\nu}}
$$

donde vemos que os elementos principais nessa definição são as componentes da matriz $\Omega^{-1}$. Além da bilinearidade, pode-se provar que os PP também satisfazem as propriedades de antissimetria, identidade de Jacobi e regra de Leibniz. Por outro lado, também se tem:

$$
\left\{\xi^{\mu} ; \xi^{\nu}\right\}=\omega^{\mu \nu} .
$$

A equação 15 é de grande importância, pois ela mostra como a operação bilinear $\{\cdot ; \cdot\}$ estabelece uma conexão direta entre as variáveis canônicas (coordenadas em $M$ ) e a inversa da forma simplética.

Posto isto, podemos concluir que no caso regular a forma simplética $\omega$ é uma peça chave para estabelecer uma estrutura de Poisson no espaço de fase.

\footnotetext{
7 Posto que estamos contemplando apenas o caso regular, $\omega$ é (por hipótese) uma forma simplética, e consequentemente nãodegenerada, como já foi mencionado.

${ }^{8}$ De fato, essas transformações deixam invariante a forma simplética $\omega$.

9 De maneira mais geral, $F$ poderia depender explicitamente do tempo, nesse caso $\dot{F} \doteq\{F ; H\}+\frac{\partial F}{\partial t}$.
}

Retomando o exemplo da lagrangiana em (3), a matriz $\Omega$ obtida em $(9)$ é claramente singular e consequentemente não podemos construir os parênteses de Poisson para esse sistema devido à inexistência da matriz inversa $\Omega^{-1}$, pois os elementos $\omega^{\mu \nu}$ não podem ser definidos.

Neste ponto é natural questionarmos se será possível equipar o espaço de fase de um sistema singular com uma estrutura de Poisson, posto que a 2 -forma $\omega$ não seja mais simplética (mas pré-simplética). Frente a essa problemática Faddeev e Jackiw (F-J) desenvolveram um método iterativo baseado no teorema de Darboux generalizado [12, o qual estabelece que, dado um espaço de fase $M$ com dimensão $2 n+R$, se o posto de uma 2 -forma fechada e degenerada $\omega$ é $2 n$, então é possível encontrar um sistema de coordenadas $\left(\left\{Q^{i}\right\}_{i=1}^{n} ;\left\{P_{i}\right\}_{i=1}^{n} ;\left\{z^{r}\right\}_{r=1}^{R}\right)$, chamadas de coordenadas de Darboux, tal que a realização matricial de $\omega$ contém um bloco $2 n \times 2 n$ da forma (8) e, nessas coordenadas, a forma pré-simplética $\omega$ pode ser escrita como em (7). Porém, $R^{\prime}<R$ das equações de movimento correspondentes as coordenadas $z^{r}$ permitem, em geral, expressar apenas $R^{\prime}<R$ delas como funções das coordenadas restantes, enquanto as outras $R-R^{\prime}$ equações de movimento serão relações que envolvem apenas as coordenadas $Q^{i}$ e $P_{i}$, o que implica que nem todas elas são realmente independentes. Depois de identificar as verdadeiras coordenadas independentes, elas podem ser tomadas como novas coordenadas unificadas $\xi$ do espaço de fase e retornar assim ao ponto inicial, mas em um espaço de fase reduzido. O procedimento anterior deve ser repetido até conseguir eliminar todas as coordenadas do tipo $z ;$ com isto, obter-se-á um espaço de fase com todas as coordenadas independentes que corresponderão aos verdadeiros graus de liberdade do sistema, obtendo assim a estrutura canônica de um sistema regular, o qual finalmente permitiria definir os Parênteses de Poisson exatamente como no caso regular.

Ainda que o método F-J seja indiscutivelmente eficiente, a implementação dele pode resultar complicada, principalmente pela dificuldade de identificar as coordenadas de Darboux em cada iteração. Entretanto, Barcelos-Neto e Wotzasek (BN-W) desenvolveram uma outra alternativa que mantém a ideia central de F-J, i. e.: conseguir que um bloco da matriz $\Omega$ contenha os graus de liberdade físicos do sistema. Mas, diferentemente do método F-J, isso não é atingido reduzindo o espaço de fase, mas estendendo-o em cada iteração sem ter que fazer uso das coordenadas de Darboux. O propósito da seguinte seção é estudar a contribuição de $\mathrm{BN}-\mathrm{W}$ que permitiu tornar mais simples a proposta original de Faddeev e Jackiw.

\section{Contribuição de Barcelos-Neto e Wotzasek (caso singular)}

Em primeiro lugar, deve ficar claro que nos sistemas singulares, o espaço de fase $M$ está equipado com uma forma pré-simplética $\omega$, o qual implica que a matriz 
associada $\Omega$ é náo-invertível; logo, se $\operatorname{dim}(M)=N$ terse-á que $N=\operatorname{posto}(\Omega)+R$. Como consequência, $\Omega$ (antissimétrica por construção) deve possuir $R$ autovetores linearmente independentes associados ao autovalor nulo:

$$
\Omega v_{r}=0 \quad(r=1, \cdots, R),
$$

usando o fato da antissimetria de $\Omega$ encontra-se que $v_{r}^{t} \Omega=0$, ou em componentes: $v_{r}^{\mu} \omega_{\mu \nu}=0$. Logo, podemos fazer o seguinte:

$$
v_{r}^{\mu} \omega_{\mu \nu} \dot{\xi}^{\nu} \stackrel{100}{=} v_{r}^{\mu} \frac{\partial H}{\partial \xi^{\mu}}=0
$$

Assim, são obtidas as seguintes $R$ equações:

$$
\Gamma_{r}(\xi)=v_{r}^{\mu} \frac{\partial H}{\partial \xi^{\mu}}=0 \quad(r=1, \cdots, R) .
$$

As funções $\Gamma_{r}$, apresentadas em (16), serão chamadas de vínculos ${ }^{10}$ pois elas deixam em evidência que nem todas as coordenadas $\xi^{\mu}$ são realmente independentes.

Considerando novamente o sistema descrito pela lagrangiana em (3), posto que a matriz $\Omega$ é singular, podemos encontrar os vínculos presentes por meio do(s) autovetor(es) associado(s) ao autovalor nulo, segundo (16). Neste caso o único autovetor resulta ser $v=$ $\left(\begin{array}{c}0 \\ 1 \\ 1-\alpha\end{array}\right)$ e com isto o vínculo buscado será dado por:

$$
\Gamma=(0) \frac{\partial H}{\partial q_{1}}+(1) \frac{\partial H}{\partial q_{2}}+(1-\alpha) \frac{\partial H}{\partial p_{1}}=0 .
$$

Assim, o vínculo resulta:

$$
\Gamma=-\alpha\left(p_{1}-q_{2}\right)+\beta\left(q_{1}-q_{2}\right)=0 .
$$

Uma vez que o caráter singular do sistema é devido à presença dos vínculos, é natural exigir que eles permaneçam inalterados enquanto o sistema evolui. Para esse fim basta impor as $R$ condições $\dot{\Gamma}_{r}=0$ à própria dinâmica do sistema. Com isso, o problema variacional para o funcional ação agora terá $R$ restrições que, em princípio, dependem das coordenadas e das velocidades; porém, uma vez que essa dependência é devida a uma derivada total (em relação à variável $t$ ), elas podem ser integradas para dar lugar a outras restrições que dependerão apenas das coordenadas.

Assim, em conformidade com o princípio de Hamilton:

$$
\delta S=\int_{t_{1}}^{t_{2}} \frac{\delta S}{\delta \xi^{\mu}(t)} \delta \xi^{\mu}(t) d t=0
$$

mas, neste caso nem todos os $N$ deslocamentos $\delta \xi^{\mu}$ são independentes, pois devem ser compatíveis com as $R$ restrições $\dot{\Gamma}_{r}=0$; consequentemente, apenas $N-R$ dos

\footnotetext{
$\overline{10}$ Posto que esses vínculos foram obtidos utilizando as equações de movimento, é lógico esperar que sejam consistentes com elas.
}

$\delta \xi^{\mu}$ serão realmente independentes. No estudo de problemas variacionais com restrições prova-se que este tipo de problema pode ser abordado de maneira equivalente fazendo uso de $R$ multiplicadores de Lagrange $\lambda^{r}=\lambda^{r}(t)$ e definindo um funcional auxiliar como segue:

$$
\tilde{S}[\xi, \lambda] \doteq \int_{t_{1}}^{t_{2}}\left(L(\xi ; \dot{\xi})-\lambda^{r}(t) \dot{\Gamma}_{r}(\xi)\right) d t .
$$

Observe-se que $\tilde{S}$ é um funcional definido sobre $N+R$ funções, a saber, $\left\{\xi^{\mu}\right\}_{\mu=1}^{N}$ e $\left\{\lambda^{r}\right\}_{r=1}^{R}$. Por outro lado, devido às restrições presentes, $R \operatorname{dos} \xi^{\mu}$ são funções das restantes, o que sugere que a extremização de $\tilde{S}$ não poderá ser realizada usando procedimento usual do caso irrestritd11 Um teorema do cálculo variacional [13] estabelece que as curvas extremais $\xi^{\mu}=\xi^{\mu}(t)$ que são soluções do problema variacional sem restrições ${ }^{12}$ também atenderão às equações de Euler-Lagrange correspondentes ao funcional auxiliar:

$$
\frac{\delta \tilde{S}}{\delta \xi^{\mu}}=0 \quad(\mu=1, \cdots N)
$$

para uma escolha apropriada dos fatores $\lambda^{r}$. Lembrando que a lagrangiana canônica é dada por (1) ter-se-á:

$$
\begin{aligned}
\tilde{S} & =\int_{t_{1}}^{t_{2}}\left(\theta_{\nu} \dot{\xi}^{\nu}-H-\dot{\Gamma}_{r} \lambda^{r}\right) d t \\
& =\int_{t_{1}}^{t_{2}}\left[\theta_{\nu} \dot{\xi}^{\nu}-H-\left(\frac{d}{d t}\left(\Gamma_{r} \lambda^{r}\right)-\Gamma_{r} \dot{\lambda}^{r}\right)\right] d t \\
& =\int_{t_{1}}^{t_{2}}\left(\theta_{\nu} \dot{\xi}^{\nu}-H+\Gamma_{r} \dot{\lambda}^{r}\right) d t,
\end{aligned}
$$

pois nos problemas de extremos fixos a contribuição dos termos de superfície é nula. Ora, uma vez que o problema variacional (irrestrito) para $\tilde{S}$ está realmente definido sobre a parte independente das coordenadas $\xi^{\mu}$, é mais apropriado escrever:

$$
\tilde{S}=\int_{t_{1}}^{t_{2}}\left(\theta_{\nu} \dot{\xi}^{\nu}+\Gamma_{r} \dot{\lambda}^{r}-\left.H\right|_{\Gamma=0}\right) d t .
$$

As equações de movimento correspondentes resultam:

$$
\left\{\begin{array}{c}
\omega_{\mu \nu} \dot{\xi}^{\nu}+\frac{\partial \Gamma_{r}}{\partial \xi^{\mu}} \dot{\lambda}^{r}-\frac{\partial}{\partial \xi^{\mu}}\left(\left.H\right|_{\Gamma=0}\right)=0 \\
-\dot{\Gamma}_{r}=0
\end{array},\right.
$$

com $\mu, \nu=1, \cdots, N$ e $r=1, \cdots, R$.

Neste ponto é relevante assinalar que em muitas ocasiões algumas das coordenadas originais $\xi^{\mu}$ não aparecem mais na função dentro da integral em $\tilde{S}$ devido a que é possível que os próprios vínculos apareçam na hamiltoniana $H$ multiplicados por algumas das coordenadas ${ }^{13}$ e, nesse caso, elas desaparecerão em $\left.H\right|_{\Gamma=0}$.

\footnotetext{
11 Isso devido a que, por suposição, as curvas paramétricas no argumento de um funcional são sempre independentes.

12 No qual todos os $\delta \xi^{\mu}$ podem ser considerados independentes.

13 Cujas velocidades correspondentes também não estejam presentes na parte $\theta_{\nu} \dot{\xi}^{\nu}$
} 
Como consequência, aquelas variáveis absorvidas não terão uma dinâmica própria, razão pela qual não deverão ser consideradas no conjunto de variáveis canônicas ${ }^{14}$ Então, se sobreviverem $N^{\prime} \leqslant N$ coordenadas, podemos rotulá-las como $\left(\zeta^{1} ; \cdots ; \zeta^{N^{\prime}}\right)$, ou $\left\{\zeta^{a}\right\}_{a=1}^{N^{\prime}}$.

Embora o funcional $\tilde{S}$ seja originalmente definido sobre as $(M+N)$ funções $\xi^{\mu}$ e $\lambda^{r}$, as variáveis que têm lugar na evolução do sistema são representadas pelas $\left(M+N^{\prime}\right)$ funções $\zeta^{a}$ e $\lambda^{r}$; então, elas podem ser tomadas como coordenadas de um novo conjunto que vamos denotar por $M^{(1)}$. Com isto, $\tilde{S}$ pode ser visto como uma ação em $M^{(1)}$ e, consequentemente, a função dentro da integral pode ser tomada como uma lagrangiana, que chamaremos $L^{(1)}$, que ademais resulta estar escrita na forma canônica (em relação às novas coordenadas), pois é linear nas velocidades em $M^{(1)}$ :

$$
L^{(1)} \doteq \vartheta_{a} \dot{\zeta}^{a}+\Gamma_{r} \dot{\lambda}^{r}-\left.H\right|_{\Gamma=0} .
$$

Lembre-se de que os coeficientes das velocidades na lagrangiana canônica são as componentes de um objeto chamado de 1-forma canônica. Então, levando em conta que as coordenadas no conjunto $M^{(1)}$ são $\left(\zeta^{1}, \cdots, \zeta^{N^{\prime}} ; \lambda^{1}, \cdots, \lambda^{R}\right)$ neste caso ter-se-á:

$$
\theta^{(1)}=\underbrace{\vartheta_{1} d \zeta^{1}+\cdots+\vartheta_{N^{\prime}} d \zeta^{N^{\prime}}}_{\vartheta(\zeta) \equiv \theta(\xi)}+\Gamma_{1} d \lambda^{1}+\cdots+\Gamma_{R} d \lambda^{R} .
$$

Com isto:

$$
\theta^{(1)}=\vartheta+\Gamma_{r} d \lambda^{r}
$$

Ou também, usando a representação como matriz linha:

$$
\Theta^{(1)}=\left(\begin{array}{llllll}
\vartheta_{1} & \cdots & \vartheta_{N} & \Gamma_{1} & \cdots & \Gamma_{R}
\end{array}\right) \stackrel{!}{=}\left(\begin{array}{ll}
\vartheta & \Gamma
\end{array}\right) .
$$

Com isto:

$$
L^{(1)}=\left(\begin{array}{ll}
\vartheta & \Gamma
\end{array}\right)\left(\begin{array}{l}
\dot{\zeta} \\
\dot{\lambda}
\end{array}\right)-\left.H\right|_{\Gamma=0}
$$

Colocando as coordenadas de $M^{(1)}$ no vetor $\xi^{(1)}=\left(\begin{array}{l}\zeta \\ \lambda\end{array}\right)$ e chamando $\left.H^{(1)} \stackrel{!}{=} H\right|_{\Gamma=0}$, a lagrangiana canônica $L^{(1)}$ se escreve simplesmente como:

$$
L^{(1)}=\theta_{\tau}^{(1)} \dot{\xi}^{(1) \tau}-H^{(1)} \quad\left(\tau=1, \cdots, N^{\prime}+R\right),
$$

que tem exatamente a mesma forma que a lagrangiana canônica apresentada em (1).

Para o exemplo da lagrangiana em (3):

- $H^{(1)}=\left.H\right|_{\Gamma=0} \stackrel{17}{=} \frac{1}{2}\left(1-\frac{\alpha^{2}}{\beta}\right)\left(p_{1}-q_{2}\right)^{2}$.

- $L^{(1)}=p_{1} \dot{q}_{1}+(1-\alpha) q_{1} \dot{q}_{2}-H^{(1)}$.

Note-se que neste caso nenhuma das variáveis canônicas originais desaparece, portanto $\zeta=\xi$.

\footnotetext{
$\overline{14}$ Mais adiante mostraremos que suprimir essas variáveis é de grande importância na presente abordagem.
}

- $\xi^{(1)}=\left(\begin{array}{c}\zeta \\ \lambda\end{array}\right) \quad \Rightarrow \quad \xi^{(1) t}=\left(\begin{array}{llll}q_{1} & q_{2} & p_{1} & \lambda\end{array}\right)$.

- $\theta^{(1)} \stackrel{19}{=} \theta+\left[-\alpha\left(p_{1}-q_{2}\right)+\beta\left(q_{1}-q_{2}\right)\right] d \lambda$.

Lembremos que o elemento chave na presente abordagem é a forma simplética (definida no espaço de fase), pois a sua inversa nos fornece a estrutura de Poisson desejada. Logo, podemos obter uma 2-forma, candidata a ser uma forma simplética em $M^{(1)}$, tomando a derivada exterior da 1 -forma canônica $\theta^{(1)}$, isto é:

$$
\begin{aligned}
\omega^{(1)} & =d \theta^{(1)}=d\left(\vartheta+\Gamma_{r^{\prime}} d \lambda^{r^{\prime}}\right)=d \vartheta+\frac{\partial \Gamma_{r^{\prime}}}{\partial \xi^{(1) \tau}} d \xi^{(1) \tau} \wedge d \lambda^{r^{\prime}} \\
& =d \vartheta+\frac{\partial \Gamma_{r^{\prime}}}{\partial \zeta^{a}} d \zeta^{a} \wedge d \lambda^{r^{\prime}}+\frac{\partial \Gamma_{r^{\prime \prime}}}{\partial \lambda^{r}} d \lambda^{r} \wedge d \lambda^{r^{\prime}} \\
& =d \vartheta+\frac{\partial \Gamma_{r^{\prime}}}{\partial \zeta^{a}} d \zeta^{a} \wedge d \lambda^{r^{\prime}} \stackrel{!}{=} \sigma+\partial_{a} \Gamma_{r^{\prime}} d \zeta^{a} \wedge d \lambda^{r^{\prime}}
\end{aligned}
$$

Assim:

$$
\omega^{(1)}=\sigma+\partial_{a} \Gamma_{r^{\prime}} d \zeta^{a} \wedge d \lambda^{r^{\prime}}
$$

Logo, ter-se-á que a matriz $\Omega^{(1)}$ resulta:

$$
\left(\begin{array}{ccc:ccc}
0 & \cdots & \sigma_{1 N^{\prime}} & \partial_{1} \Gamma_{1} & \cdots & \partial_{1} \Gamma_{R} \\
\vdots & \ddots & \vdots & \vdots & \ddots & \vdots \\
-\sigma_{1 N^{\prime}} & \cdots & 0 & \partial_{N^{\prime}} \Gamma_{1} & \cdots & \partial_{N^{\prime}} \Gamma_{R} \\
\hdashline-\bar{\partial}_{1} \bar{\Gamma}_{1} & \cdots & -\partial_{N^{\prime}} \Gamma_{1} & 0 & \cdots & 0 \\
\vdots & \ddots & \vdots & \vdots & \ddots & \vdots \\
-\partial_{1} \Gamma_{R} & \cdots & -\partial_{N^{\prime}} \Gamma_{R} & 0 & \cdots & 0
\end{array}\right)
$$

ou de maneira mais compacta, $\Omega^{(1)}=\left(\begin{array}{c:c}\Sigma & \partial \Gamma \\ \hdashline-\bar{\partial} \Gamma & 0\end{array}\right)$. Neste ponto é importante fazer um comentário sobre a relação entre as 1 -formas $\theta$ e $\vartheta$, as 2 -formas $\omega=d \theta$ e $\sigma=d \vartheta$, e as representações matriciais destas últimas, $\Omega$ e $\Sigma$. Por construção, $\vartheta$ é essencialmente idêntica a $\theta$, mas definida sobre uma base reduzida na qual as coordenadas que não aparecem mai: 15 em $L^{(1)}$ foram suprimidas. Por exemplo, seja a lagrangiana

$$
L^{(1)}=\theta_{2} \dot{\xi}^{2}+\cdots+\theta_{N} \dot{\xi}^{N}+\Gamma_{r} \dot{\lambda}^{r}-H^{(1)},
$$

com $\theta_{2}, \cdots, \theta_{N}, \Gamma_{r}, H$ funções que independem de $\xi^{1}$. Nesse caso:

$$
\begin{aligned}
& \text { - } \theta=(0) d \xi^{1}+\theta_{2} d \xi^{2}+\cdots \theta_{N} d \xi^{N} \text {. } \\
& \text { - } \omega=\sum_{\mu<\nu} \omega_{\mu \nu} d \xi^{\mu} \wedge d \xi^{\nu} \\
& =\sum_{\nu} \omega_{1 \nu} d \xi^{1} \wedge d \xi^{\nu}+\sum_{1<\mu<\nu} \omega_{\mu \nu} d \xi^{\mu} \wedge d \xi^{\nu} \\
& \operatorname{com} \omega_{1 \nu}=\frac{\partial \theta_{\nu}}{\partial \xi^{1}}-\frac{\partial(0)}{\partial \xi^{\nu}}=0 .
\end{aligned}
$$

$\overline{15}$ Nem as velocidades correspondentes a essas coordenadas. 


$$
\text { - } \Omega=\left(\begin{array}{ccccc}
0 & 0 & 0 & \cdots & 0 \\
0 & 0 & \omega_{23} & \cdots & \omega_{2 N} \\
0 & -\omega_{23} & 0 & \cdots & \omega_{3 N} \\
\vdots & \vdots & \vdots & \ddots & \vdots \\
0 & -\omega_{2 N} & -\omega_{3 N} & \cdots & 0
\end{array}\right) \text {. }
$$

Como podemos ver, a total ausência de $\xi^{1}$ traz como consequência que a matriz $\Omega$ contenha apenas zeros tanto na linha quanto na coluna correspondentes a $\xi^{1}$. Definindo o conjunto das coordenadas $\zeta^{a}$ (sem considerar $\left.\xi^{1}\right)$ terse-á:

- $\left(\zeta^{1} ; \cdots ; \zeta^{N-1}\right) \stackrel{!}{=}\left(\xi^{2} ; \cdots ; \xi^{N}\right)$.

- $\vartheta=\vartheta_{a} d \zeta^{a}=\sum_{\nu>1} \theta_{\nu} d \xi^{\nu}$.

- $\sigma=\sum_{a<b} \sigma_{a b} d \zeta^{a} \wedge d \zeta^{b}=\sum_{1<\mu<\nu} \omega_{\mu \nu} d \xi^{\mu} \wedge d \xi^{\nu}$.

- $\Sigma=\left(\begin{array}{cccc}0 & \omega_{23} & \cdots & \omega_{2 N} \\ -\omega_{23} & 0 & \cdots & \omega_{3 N} \\ \vdots & \vdots & \ddots & \vdots \\ -\omega_{2 N} & -\omega_{3 N} & \cdots & 0\end{array}\right)$.

Daqui, podemos ver que a matriz $\Sigma$ não é nada mais que a matriz $\Omega$, mas sem as linhas e colunas que contêm apenas zeros devido à ausência de algumas coordenadas. A principal importância de trabalhar com $\Sigma$ em vez de $\Omega$ é o fato de que, devido à independência dos vínculos $\Gamma_{r}$ das coordenadas que sumiram, as derivadas dos vínculos em relação a essas coordenadas serão sempre 0 , o que traria como consequência que, devido a 20 , no caso de usar a matriz $\Omega$ (e não $\Sigma$ ), a nova matriz $\Omega^{(1)}$ teria também linhas e colunas contendo apenas zeros nas mesmas posições que $\Omega$ e seria, evidentemente, ainda singular.

Ora, observa-se que a matriz $\Omega^{(1)}$ contém a matriz $\Sigma$ como um bloco das primeiras $N^{\prime}$ linhas e $N^{\prime}$ colunas, que correspondem às verdadeiras ${ }^{16}$ variáveis canônicas do espaço de fase original $M$.

Em relação ao exemplo da lagrangiana em (3), podemos obter a matriz $\Omega^{(1)}$ a partir da 2 -forma $\omega^{(1)}=d \theta^{(1)}$ como segue:

- $\omega^{(1)}=\omega-\alpha d p_{1} \wedge d \lambda+\alpha d q_{2} \wedge d \lambda+\beta d q_{1} \wedge d \lambda$ $-\beta d q_{2} \wedge d \lambda$.

- A realização matricial será:

$$
\Omega^{(1)}=\left(\begin{array}{ccc:c}
0 & 1-\alpha & -1 & \beta \\
-1+\alpha & 0 & 0 & \alpha-\beta \\
1 & 0 & 0 & -\alpha \\
\hdashline-\bar{\beta}-\alpha+\beta & \alpha & 0
\end{array}\right) .
$$

É importante observar a compatibilidade da dinâmica das quantidades com o índice superior (1), que correspondem à primeira ordem de iteração no método F-J, com as equações de movimento apresentadas em (18). Uma vez que a lagrangiana $L^{(1)}$ está na forma

\footnotetext{
${ }^{16}$ No sentido que possuem uma dinâmica própria.
}

canônica, seguindo o procedimento apresentado na seção 2 as equações de movimento correspondentes serão:

$$
\omega_{\rho \tau}^{(1)} \dot{\xi}^{(1) \tau}=\frac{\partial H^{(1)}}{\partial \xi^{(1) \rho}},
$$

em que $\omega_{\rho \tau}^{(1)}$ sejam as componentes da 2 -forma $\omega^{(1)}=$ $d \theta^{(1)}$ na base $\left\{d \xi^{(1) \rho} \wedge d \xi^{(1) \tau}\right\}$, e portanto:

$$
\omega_{\rho \tau}^{(1)}=\frac{\partial \theta_{\tau}^{(1)}}{\partial \xi^{(1) \rho}}-\frac{\partial \theta_{\rho}^{(1)}}{\partial \xi^{(1) \tau}} .
$$

Fazendo $\rho=(a, r)$ e $\tau=\left(b, r^{\prime}\right)$ e levando em conta que $\Theta^{(1)}=\left(\begin{array}{ll}\vartheta & \Gamma\end{array}\right)$ e $\xi^{(1)}=\left(\begin{array}{l}\zeta \\ \lambda\end{array}\right)$, pode-se verificar rapidamente que os $\omega_{\rho \tau}^{(1)}$ são, com efeito, os elementos da matriz $\Omega^{(1)}$. Usando essa mesma rotulação nas equações de movimento em $(22)$ se obtém:

$$
\sigma_{a b} \dot{\zeta}^{b}+\frac{\partial \Gamma_{r^{\prime}}}{\partial \zeta^{a}} \dot{\lambda}^{r^{\prime}}=\frac{\partial H^{(1)}}{\partial \zeta^{a}}, \quad-\frac{\partial \Gamma_{r}}{\partial \zeta^{b}} \dot{\zeta}^{b}=0 .
$$

Podemos ver que, com efeito, estas equações coincidem com as apresentadas em (18). Lembremos que as variáveis $\zeta^{a}$ estão incluídas dentro das variáveis $\xi^{\mu}$. Chamemse $\eta^{i}$ as coordenadas restantes (que seriam aquelas que são absorvidas ao passar à primeira ordem de iteração no algoritmo F-J), ou seja:

$$
\left\{\xi^{\mu}\right\}_{\mu=1}^{N}=\left\{\zeta^{a}\right\}_{a=1}^{N^{\prime}} \cup\left\{\eta^{i}\right\}_{i=1}^{N-N^{\prime}},
$$

o que nos permite fazer as rotulações $\mu=(a, i)$ e $\nu=$ $(b, j)$, com isto, as equações em (18) podem ser escritas como:

$$
\left\{\begin{array}{c}
\omega_{a b} \dot{\zeta}^{b}+\omega_{a j} \dot{\eta}^{j}+\frac{\partial \Gamma_{r}}{\partial \zeta^{a}} \dot{\lambda}^{r}-\frac{\partial H^{(1)}}{\partial \zeta^{a}}=0 \\
\omega_{i b} \dot{\zeta}^{b}+\omega_{i j} \dot{\eta}^{j}+\frac{\partial \Gamma_{r}}{\partial \eta^{i}} \dot{\eta}^{i}-\frac{\partial H^{(1)}}{\partial \eta^{i}}=0 \\
-\dot{\Gamma}_{r}=-\frac{\partial \Gamma_{r}}{\partial \xi^{\nu}} \dot{\xi}^{\nu}=-\frac{\partial \Gamma_{r}}{\partial \zeta^{b}} \dot{\zeta}^{b}-\frac{\partial \Gamma_{r}}{\partial \eta^{j}} \dot{\eta}^{j}=0
\end{array}\right.
$$

Mas, levando em conta que:

$$
\omega_{a b}=\sigma_{a b}, \quad \omega_{a i}=0=\omega_{i j}, \quad \frac{\partial \Gamma_{r}}{\partial \eta^{i}}=0=\frac{\partial H^{(1)}}{\partial \eta^{i}} ;
$$

as equações obtidas resultam:

$$
\left\{\begin{aligned}
\sigma_{a b} \dot{\zeta}^{b}+\frac{\partial \Gamma_{r}}{\partial \zeta^{a}} \dot{\lambda}^{r}-\frac{\partial H^{(1)}}{\partial \zeta^{a}} & =0 \\
0 & =0 \\
-\frac{\partial \Gamma_{r}}{\partial \zeta^{b}} \dot{\zeta}^{b} & =0
\end{aligned}\right.
$$

que são as mesmas equações que em 23.

Assim, vemos que as quantidades em primeira ordem de iteração (definidas no conjunto $M^{(1)}$ ) mantêm a mesma estrutura canônica da formulação de FaddeevJackiw. Este último fato nos permite afirmar que se a 
2-forma $\omega^{(1)}$ resultasse ser simplética, a inversa dela permitiria definir uma estrutura de Poisson em $M^{(1)}$. Contudo, os únicos parênteses de Poisson que serão de interesse físico serão os correspondentes às coordenadas $\xi^{\mu}$, pois as outras coordenadas são essencialmente os multiplicadores de Lagrange inseridos para considerar os vínculos. Desta forma, se a matriz $\Omega^{(1)}$ for invertível, apenas devemos interessar-nos pelo bloco das primeiras $N^{\prime}$ linhas e $N^{\prime}$ colunas da sua inversa.

Para o exemplo da lagrangiana em (3), o determinante da matriz $\Omega^{(1)}$ é $\left(\alpha^{2}-\beta\right)^{2}$. Então, sob o suposto de que $\alpha^{2} \neq \beta$, ela será não-singular com inversa:

$$
\left[\Omega^{(1)}\right]^{-1}=\frac{1}{\alpha^{2}-\beta}\left(\begin{array}{ccc:c}
0 & \alpha & \alpha-\beta & 0 \\
-\alpha & 0 & -\beta & -1 \\
\hdashline \alpha+\beta & \beta & 0 & \alpha-1 \\
\hdashline 0 & -\alpha-\overline{1} & \overline{1}-1
\end{array}\right) .
$$

Com isto, já podemos apresentar os PP das variáveis canônicas originais por meio de (15):

$$
\begin{aligned}
& \text { - }\left\{\xi^{(1) 1} ; \xi^{(1) 2}\right\}=\omega^{(1) 12} \Rightarrow\left\{q_{1} ; q_{2}\right\}=\frac{\alpha}{\alpha^{2}-\beta} . \\
& \text { - }\left\{\xi^{(1) 1} ; \xi^{(1) 3}\right\}=\omega^{(1) 13} \Rightarrow\left\{q_{1} ; p_{1}\right\}=\frac{\alpha-\beta}{\alpha^{2}-\beta} . \\
& \text { - }\left\{\xi^{(1) 2} ; \xi^{(1) 3}\right\}=\omega^{(1) 23} \Rightarrow\left\{q_{2} ; p_{1}\right\}=\frac{-\beta}{\alpha^{2}-\beta} .
\end{aligned}
$$

Poderia também dar-se o caso de que a matriz $\Omega^{(1)}$ não seja invertível, e, por conseguinte, $\omega^{(1)}$ não será uma forma simplética. Nesta situação o procedimento todo deverá ser repetido, mas partindo das quantidades obtidas na primeira iteração para passar à segunda iteração. Em geral, esperamos que após um certo número $n$ (finito) de iterações, se obtenha uma forma simplética $\omega^{(n)}$ cuja inversa dê lugar à estrutura de Poisson (no conjunto $M^{(n)}$ ) desejada.

Embora, em princípio, já temos um algoritmo para obter os PP para qualquer sistema (regular ou singular), nosso objetivo final sempre será a dinâmica do sistema, que é determinada a partir das equações de movimento. Como já foi apresentado na subseção 2.1. podemos obter a evolução temporal de qualquer função dinâmica por meio de (13), em que o gerador da evolução é a hamiltoniana definida no espaço de fase correspondente. $\mathrm{Na}$ presente abordagem, o gerador de evolução temporal resulta ser a hamiltoniana $H^{(n)}$, que corresponde à ordem de iteração do algoritmo F-J que permitiu obter a estrutura de Poisson, isso devido a que ela já é compatível com todos os vínculos que foram encontrados até a $n$-ésima etapa do procedimento.

Mais uma vez, para o exemplo da lagrangiana em (3) o nosso gerador de evolução temporal será a hamiltoniana $H^{(1)}$ e com ela podemos obter as equações de movimento para as variáveis canônicas. Por exemplo, para $q_{1}$ ter-se-á:

$$
\dot{q}_{1}=\left\{q_{1} ; H^{(1)}\right\}=\left\{q_{1} ; \frac{1}{2}\left(1-\frac{\alpha^{2}}{\beta}\right)\left(p_{1}-q_{2}\right)\right\}^{2}
$$

$$
\begin{aligned}
& =\left(1-\frac{\alpha^{2}}{\beta}\right)\left\{q_{1} ; p_{1}-q_{2}\right\}\left(p_{1}-q_{2}\right) \\
& =\left(1-\frac{\alpha^{2}}{\beta}\right)\left(\left\{q_{1} ; p_{1}\right\}-\left\{q_{1} ; q_{2}\right\}\right)\left(p_{1}-q_{2}\right) \\
& =\left(1-\frac{\alpha^{2}}{\beta}\right)\left(\frac{-\beta}{\alpha^{2}-\beta}\right)\left(p_{1}-q_{2}\right)=p_{1}-q_{2} .
\end{aligned}
$$

Similarmente se obtém:

$$
\dot{q}_{2}=\frac{\beta}{\alpha}\left(q_{1}-q_{2}\right), \quad \dot{p}_{1}=\frac{\beta}{\alpha}\left(q_{1}-q_{2}\right) .
$$

\section{Exemplos}

\subsection{Exemplo 1}

Considere-se o caso de uma partícula imersa em uma região espacial bidimensional (que será representada pelo plano $x^{1} x^{2}$ ) com um campo magnético externo. Em geral, dados os potenciais escalar $A^{0}$ e vetorial $\vec{A}$, que são funções da posição (e possivelmente do tempo), a função lagrangiana vem dada segundd

$$
L=\frac{1}{2} \dot{\vec{x}}^{2}+\dot{\vec{x}} \cdot \vec{A}-A^{0}=\frac{1}{2} \delta_{i j} \dot{x}^{i} \dot{x}^{j}+\delta_{i j} \dot{x}^{i} A^{j}-A^{0} .
$$

Os momentos canônicos conjugados resultam:

$$
p_{i}=\frac{\partial L}{\partial \dot{x}_{i}}=\delta_{i j}\left(\dot{x}^{j}+A^{j}\right) ; \quad i, j=1,2 .
$$

Daqui podemos expressar as velocidades em termos das coordenadas e os momentos 18 .

$$
\delta^{k i} p_{i}=\delta^{k i} \delta_{i j}\left(\dot{x}^{j}+A^{j}\right)=\delta_{j}^{k}\left(\dot{x}^{j}+A^{j}\right)=\dot{x}^{k}+A^{k} .
$$

Assim:

$$
\dot{x}^{i}=\delta^{i j} p_{j}-A^{i} .
$$

Com isto, a hamiltoniana resulta:

$$
\begin{aligned}
H & =p_{i} \dot{x}^{i}-\frac{1}{2} \delta_{i j} \dot{x}^{i} \dot{x}^{j}-\delta_{i j} \dot{x}^{i} A^{j}+A^{0} \\
& =\dot{x}^{i}\left[p_{i}-\delta_{i j}\left(\frac{1}{2} \dot{x}^{j}+A^{j}\right)\right]+A^{0} \\
& =\dot{x}^{i}\left[p_{i}-\delta_{i j}\left(\frac{1}{2}\left(\delta^{j k} p_{k}-A^{j}\right)+A^{j}\right)\right]+A^{0} \\
& =\left(\delta^{i k} p_{k}-A^{i}\right)\left(\frac{1}{2} p_{i}-\frac{1}{2} \delta_{i j} A^{j}\right)+A^{0} \\
& =\frac{1}{2}\left(\delta^{i k} p_{k}-A^{i}\right)\left(\delta_{i j} \delta^{j \ell} p_{\ell}-\delta_{i j} A^{j}\right)+A^{0} \\
& =\frac{1}{2} \delta_{i j}\left(\delta^{i k} p_{k}-A^{i}\right)\left(\delta^{j \ell} p_{\ell}-A^{j}\right)+A^{0} .
\end{aligned}
$$

A seguir, suponha-se que os potenciais $A^{0}$ e $\vec{A}$ independem do tempo (explicitamente) e que o campo vetorial $\vec{A}$ tem simetria de rotação. Nesse caso ter-se-á:

\footnotetext{
17 Por uma questão de comodidade estamos considerando $m=1$ e $e=1$.

18 Não devemos confundir as componentes $\delta_{i j}$ do tensor métrico euclidiano (que determina o produto interno) com a delta de Kronecker $\delta_{j}^{i}$.
} 
- Potencial escalar:

$$
A^{0}=A^{0}(r), \text { com } r \stackrel{!}{=}|\vec{x}| .
$$

- Potencial vetorial:

$$
\vec{A}=A(r) \vec{x}_{\perp}, \operatorname{com} \vec{x}_{\perp} \stackrel{!}{=} \hat{e}_{1} x^{2}-\hat{e}_{2} x^{1} .
$$

Então: $\quad A^{1}=x^{2} A(r), \quad A^{2}=-x^{1} A(r)$

Assim, em geral podemos escrever:

$$
A^{i}=\left(\delta_{1}^{i} x^{2}-\delta_{2}^{i} x^{1}\right) A(r)=\underbrace{\left(\delta_{1}^{i} \delta_{j}^{2}-\delta_{2}^{i} \delta_{j}^{1}\right)}_{\epsilon^{i}{ }_{j}=\delta^{i k} \epsilon_{k j}} x^{j} A(r) .
$$

Escolhendo $A(r)=-\frac{B}{2}$, com $B>0$ constante, o campo magnético ${ }^{19} B^{3}$ será dado por:

$$
\begin{aligned}
B^{3} & =\epsilon^{i k} \delta_{k j} \partial_{i}\left(\delta^{j \ell} \epsilon_{\ell m} x^{m} A(r)\right)=\epsilon^{i k} \delta_{k}^{\ell} \epsilon_{\ell m} \partial_{i} x^{m} A(r) \\
& =\epsilon^{i k} \epsilon_{k m} \partial_{i} x^{m} A(r)=-\epsilon^{k i} \epsilon_{k m} \partial_{i} x^{m} A(r) \\
& =-\delta_{m}^{i} \partial_{i} x^{m} A(r)=-\partial_{i} x^{i} A(r)=-2 A(r)=B .
\end{aligned}
$$

Assim, sob esta consideração o campo magnético resulta ser uniforme.

Adicionalmente, escolhemos um potencial escalar da forma $A^{0}=\frac{1}{2} k \vec{x}^{2}$. Com todas as considerações anteriores a lagrangiana resulta:

$$
L=\frac{1}{2} \dot{\vec{x}}^{2}-\frac{B}{2} \dot{\vec{x}} \cdot \vec{x}_{\perp}-\frac{1}{2} k \vec{x}^{2} .
$$

Agora observe-se o seguinte:

$$
\dot{\vec{x}} \cdot \vec{x}_{\perp}=\dot{x}^{1} x^{2}-\dot{x}^{2} x^{1} \stackrel{!}{=} \dot{\vec{x}} \times \vec{x},
$$

com isto, a lagrangiana (24) pode ser escrita como:

$$
\begin{aligned}
L & =\frac{1}{2} \dot{\vec{x}}^{2}-\frac{B}{2} \dot{\vec{x}} \times \vec{x}-\frac{1}{2} k \vec{x}^{2} \\
& =\frac{1}{2} \delta_{i j} \dot{x}^{i} \dot{x}^{j}-\frac{B}{2} \epsilon_{i j} \dot{x}^{i} x^{j}-\frac{k}{2} \delta_{i j} x^{i} x^{j} .
\end{aligned}
$$

Os momentos canônicos conjugados serão:

$$
p_{i}=\delta_{i j} \dot{x}^{j}-\frac{B}{2} \epsilon_{i j} x^{j} \Rightarrow \dot{x}^{i}=\delta^{i j}\left(p_{j}+\frac{B}{2} \epsilon_{j k} x^{k}\right) .
$$

Também, a hamiltoniana correspondente resulta:

$$
H=\frac{1}{2} \delta^{i j}\left(p_{i}+\frac{B}{2} \epsilon_{i k} x^{k}\right)\left(p_{j}+\frac{B}{2} \epsilon_{j k} x^{k}\right)+\frac{k}{2} \delta_{i j} x^{i} x^{j} .
$$

Uma vez que é possível expressar todas as velocidades em termos das coordenadas e dos momentos, o sistema em consideração é regular. Entretanto, é possível obter um sistema singular a partir do anterior fazendo $m \rightarrow 0$ 14. (o que equivale a desconsiderar o termo cinético na lagrangiana original), resultando:

19 Rigorosamente $\vec{B}=\nabla \times \vec{A}=\hat{e}_{3}\left(\partial_{1} A^{2}-\partial_{2} A^{1}\right)$, que é um campo vetorial na direção do eixo $x^{3}$, mas como possui apenas uma componente, se lhe associa a quantidade escalar $B^{3}=\partial_{1} A^{2}-$ $\partial_{2} A^{1}=\left(\delta_{1}^{i} \delta_{j}^{2}-\delta_{2}^{i} \delta_{j}^{1}\right) \partial_{i} A^{j}=\epsilon^{i}{ }_{j} \partial_{i} A^{j}=\epsilon^{i k} \delta_{k j} \partial_{i} A^{j}$. De fato, geralmente é usada a seguinte notação para o produto vetorial de dois vetores sobre o plano $x^{1} x^{2}: \vec{u} \times \vec{v} \stackrel{!}{=} u^{1} v^{2}-u^{2} v^{1}=\epsilon_{i j} u^{i} v^{j}$.
- Lagrangiana:

$$
L=-\frac{B}{2} \dot{\vec{x}} \times \vec{x}-\frac{1}{2} k \vec{x}^{2}=-\frac{B}{2} \epsilon_{i j} \dot{x}^{i} x^{j}-\frac{k}{2} \delta_{i j} x^{i} x^{j} .
$$

- Momentos canônicos conjugados:

$$
p_{i}=-\frac{B}{2} \epsilon_{i j} x^{j} .
$$

- Hamiltoniana:

$$
H=\frac{1}{2} k \vec{x}^{2}=\frac{k}{2} \delta_{i j} x^{i} x^{j} .
$$

Observe-se que a lagrangiana é uma função de primeira ordem nas velocidades $\dot{x}^{i}$; além disso, os momentos canônicos $p_{i}$ são funções das coordenadas $x^{i}$ e devido a isso, as 1 -formas $d x^{i}$ e $d p_{i}$ não serão independentes. Por outro lado, em conformidade com a formulação F-J, essa lagrangiana já está na forma canônica em relação às variáveis $x^{i}$, pois ela tem a forma:

$$
L(\vec{x} ; \dot{\vec{x}})=\theta_{i}(\vec{x}) \dot{x}^{i}-H(\vec{x}), \quad \text { com: } \quad \theta_{i}=-\frac{B}{2} \epsilon_{i j} x^{j} .
$$

Então, nessas coordenadas, a 1-forma canônica resulta ser $\theta=\theta_{i} d x^{i}$. Logo, tomando a derivada exterior, se obtém a 2 -forma:

$$
\omega=d \theta=-\frac{B}{2} \epsilon_{i j} d x^{j} \wedge d x^{i}=\frac{B}{2} \epsilon_{i j} d x^{i} \wedge d x^{j} .
$$

Levando em conta que $\omega=\frac{1}{2} \omega_{i j} d x^{i} \wedge d x^{j}$, encontra-se que os elementos da matriz associada à 2 -forma $\omega$ são $\omega_{i j}=B \epsilon_{i j}$. Explicitamente a matriz envolvida é:

$$
\Omega=B\left(\begin{array}{cc}
0 & 1 \\
-1 & 0
\end{array}\right)
$$

a qual é invertível e, consequentemente, $\omega$ resulta ser uma forma simplética cuja inversa permitirá obter diretamente os parênteses de Poisson das variáveis dinâmicas escolhidas:

$$
\begin{aligned}
\omega_{i j}=B \epsilon_{i j} \Rightarrow B \omega^{k i} \epsilon_{i j} & =\omega^{k i} \omega_{i j}=\delta_{j}^{k} \\
B \omega^{k i} \epsilon_{i j} \epsilon^{\ell j} & =\delta_{j}^{k} \epsilon^{\ell j} \\
B \omega^{k i} \delta_{i}^{\ell} & =\epsilon^{\ell k} \Rightarrow \omega^{k \ell}=\frac{1}{B} \epsilon^{\ell k} .
\end{aligned}
$$

Assim:

$$
\omega^{i j}=-\frac{1}{B} \epsilon^{i j} \Rightarrow\left\{x^{i} ; x^{j}\right\}=-\frac{1}{B} \epsilon^{i j} .
$$

Mais ainda, uma vez que os momentos canônicos $p_{i}$ são funções das coordenadas $x^{i}$, também pode-se obter os parênteses de Poisson que envolvem os $p_{i}$ :

$$
\begin{aligned}
-\left\{x^{i} ; p_{j}\right\} & =\left\{x^{i} ;-\frac{B}{2} \epsilon_{j k} x^{k}\right\}=-\frac{B}{2} \epsilon_{j k}\left\{x^{i} ; x^{k}\right\} \\
& =-\frac{B}{2} \epsilon_{j k}\left(-\frac{1}{B}\right) \epsilon^{i k}=\frac{1}{2} \epsilon_{j k} \epsilon^{i k}=\frac{1}{2} \delta_{j}^{i} . \\
\text { - }\left\{p_{i} ; p_{j}\right\} & =\left\{-\frac{B}{2} \epsilon_{i k} x^{k} ;-\frac{B}{2} \epsilon_{j \ell} x^{\ell}\right\} \\
& =\frac{B^{2}}{4} \epsilon_{i k} \epsilon_{j \ell}\left\{x^{k} ; x^{\ell}\right\}=\frac{B^{2}}{4} \epsilon_{i k} \epsilon_{j \ell}\left(-\frac{1}{B} \epsilon^{k \ell}\right) \\
& =-\frac{B}{4} \epsilon_{i k} \delta_{j}^{k}=-\frac{B}{4} \epsilon_{i j} .
\end{aligned}
$$




\subsection{Exemplo 2}

Considere-se o caso de uma partícula movendo-se em um espaço de configuração 3-dimensional, em conformidade com a seguinte função lagrangiana [15]:

$$
L=\left(q_{2}+q_{3}\right) \dot{q}_{1}+k \dot{q}_{3}+\frac{1}{2}\left(k^{2}-2 q_{2} q_{3}-q_{3}^{2}\right),
$$

com $k$ constante. Os momentos canônicos conjugados às coordenadas $q_{i}$ serão:

$$
p_{1}=q_{2}+q_{3}, \quad p_{2}=0, \quad p_{3}=k .
$$

Com isto, a hamiltoniana resulta:

$$
H=-\frac{1}{2}\left(k^{2}-2 q_{2} q_{3}-q_{3}^{2}\right)
$$

Vê-se que essa lagrangiana é linear nas velocidades e, consequentemente, já está em sua forma canônica; então, se escolhem como coordenadas do espaço de fase: $\xi=\left(\begin{array}{l}q_{1} \\ q_{2} \\ q_{3}\end{array}\right)$. Assim, identifica-se que a 1 -forma canônica resulta ser:

$$
\theta=\left(q_{2}+q_{3}\right) d q_{1}+0 d q_{2}+k d q_{3} \equiv\left(q_{2}+q_{3} \quad 0 \quad k\right) .
$$

Logo, tomando a derivada exterior, define-se a 2-forma:

$$
\omega=d \theta=d q_{2} \wedge d q_{1}+d q_{3} \wedge d q_{1} \equiv\left(\begin{array}{ccc}
0 & -1 & -1 \\
1 & 0 & 0 \\
1 & 0 & 0
\end{array}\right) .
$$

Evidentemente a matriz associada $\Omega$ é singular e tem o seguinte autovetor associado ao autovalor nulo:

$$
v=\left(\begin{array}{r}
0 \\
-1 \\
1
\end{array}\right)
$$

Logo, em conformidade com o método F-J, constrói-se o seguinte vínculo:

$\Gamma=v^{\mu} \frac{\partial H}{\partial \xi^{\mu}}=-\frac{\partial H}{\partial q_{2}}+\frac{\partial H}{\partial q_{3}}=-q_{3}+\left(q_{2}+q_{3}\right)=q_{2}=0$.

O seguinte passo será construir as quantidades correspondentes à primeira ordem de iteração:

- Hamiltoniana:

$$
H^{(1)}=\left.H\right|_{\Gamma=0}=-\frac{1}{2}\left(k^{2}-q_{3}^{2}\right) .
$$

- Lagrangiana canônica:

$$
\begin{aligned}
L^{(1)} & =\theta_{\nu} \dot{\xi}^{\nu}+\Gamma \dot{\lambda}-H^{(1)} \\
& =\left(q_{2}+q_{3}\right) \dot{q}_{1}+k \dot{q}_{3}+q_{2} \dot{\lambda}+\frac{1}{2}\left(k^{2}-q_{3}^{2}\right) .
\end{aligned}
$$

- Coordenadas ${ }^{20}$ do espaço de fase $M^{(1)}$ :

$$
\xi^{(1)}=\left(\begin{array}{c}
\zeta \\
\lambda
\end{array}\right) \quad \Rightarrow \quad \xi^{(1) t}=\left(\begin{array}{llll}
q_{1} & q_{2} & q_{3} & \lambda
\end{array}\right) .
$$

- 1-forma canônica:

$$
\theta^{(1)}=\theta+\Gamma d \lambda=\theta+q_{2} d \lambda
$$

- Derivada exterior da 1-forma canônica:

$$
\omega^{(1)}=d \theta^{(1)}=\omega+d q_{2} \wedge d \lambda .
$$

A matriz associada à 2 -forma $\omega^{(1)}$ resulta:

$$
\Omega^{(1)}=\left(\begin{array}{ccc:c}
0 & -1 & -1 & 0 \\
1 & 0 & 0 & 1 \\
1 & 0 & 0 & 0 \\
\hdashline 0 & -1 & 0 & 0
\end{array}\right) .
$$

Não é difícil ver que $\operatorname{det}\left(\Omega^{(1)}\right)=1 \neq 0 \mathrm{e}$, portanto, $\Omega^{(1)}$ é regular. Logo, a matriz inversa resulta:

$$
\left[\Omega^{(1)}\right]^{-1}=\left(\begin{array}{ccc:c}
0 & 0 & 1 & 0 \\
0 & 0 & 0 & -1 \\
-1 & 0 & 0 & 1 \\
\hdashline 0 & 1 & -1 & 0
\end{array}\right) .
$$

Uma vez que $\omega^{(1)}$ resulta ser uma forma simplética (devido à sua não-degenerescência), podemos definir uma estrutura de Poisson no espaço de fase $M^{(1)}$ segundo $\left\{\xi^{(1) \alpha} ; \xi^{(1) \beta}\right\}=\omega^{(1) \alpha \beta}$. Neste ponto é preciso lembrar que a variável auxiliar $\lambda$ foi introduzida (como multiplicador de Lagrange) apenas para impor a preservação do vínculo $\Gamma$ durante a evolução do sistema e, consequentemente, não é de interesse físico. Assim, os parênteses de Poisson das variáveis dinâmicas originais serão dadas pelo bloco constituído pelas três primeiras linhas e colunas de $\left[\Omega^{(1)}\right]^{-1}$.

$$
\left\{q_{1} ; q_{3}\right\}=1 \quad \Rightarrow \quad\left\{q_{1} ; p_{1}\right\}=\left\{q_{1} ; q_{2}+q_{3}\right\}=1 \text {. }
$$

Vale considerar que o vínculo obtido em 25 fixa a coordenada $q_{2}$ e em consequência ela não tem dinâmica. Pode-se notar que isso está em perfeita conformidade com as equações de movimento:

- $\frac{\delta S}{\delta q_{1}}=\dot{q}_{2}+\dot{q}_{3}=0$.

- $\frac{\delta S}{\delta q_{2}}=\dot{q}_{1}-q_{3}=0$.

- $\frac{\delta S}{\delta q_{3}}=\dot{q}_{1}-q_{2}-q_{3}=0$.

20 Lembre-se de que em um caso geral nem todas coordenadas originais $\xi^{\mu}$ aparecem em $L^{(1)}$; devido a isso, as variáveis remanescentes são rotuladas por $\zeta^{a}$. Porém, quando nenhuma das variáveis é absorvida tem-se que $\zeta=\xi$. 
Das equações de movimento para $q_{2}$ e $q_{3}$, observa-se que, com efeito, $q_{2}=0$. Isso sugere que uma melhor escolha para as coordenadas do espaço de fase $M^{(1)}$ seria $\xi^{(1)}=$ $\left(\begin{array}{l}q_{1} \\ q_{3}\end{array}\right)$. Com isto, as funções hamiltoniana e lagrangiana em primeira ordem de iteração serão:

$$
\begin{array}{ll}
\text { - } H^{(1)}=\left.H\right|_{M^{(1)}}=-\frac{1}{2}\left(k^{2}-q_{3}^{2}\right) . \\
\text { - } L^{(1)}=\left.L\right|_{M^{(1)}}=q_{3} \dot{q}_{1}+k \dot{q}_{3}+\frac{1}{2}\left(k^{2}-q_{3}^{2}\right) .
\end{array}
$$

Logo, a 1-forma canônica 1-iterada e a derivada exterior dela serão:

$$
\theta^{(1)}=q_{3} d q_{1}+k d q_{3}, \quad \omega^{(1)}=d q_{3} \wedge d q_{1} \equiv\left(\begin{array}{cc}
0 & -1 \\
1 & 0
\end{array}\right) .
$$

A matriz $\Omega^{(1)}$ é regular e tem por inversa $\left[\Omega^{(1)}\right]^{-1}=$ $\left(\begin{array}{cc}0 & 1 \\ -1 & 0\end{array}\right)$. Com isto, os parênteses de Poisson das variáveis dinâmicas $\xi^{(1)}$ seriam:

$$
\left\{q_{1} ; q_{3}\right\}=1
$$

que é consistente com o resultado obtido em 26. Assim, podemos concluir que no caso de haver algum vínculo que fixe alguma das variáveis dinâmicas do espaço de fase, podemos passar à seguinte ordem de iteração apenas restringindo a lagrangiana e a hamiltoniana às novas coordenadas consistentes com dito vínculo, sem a necessidade de incluir alguma variável auxiliar (multiplicador de Lagrange).

\subsection{Exemplo 3}

Considere-se um espaço de configuração 4-dimensional descrito pelas coordenadas $\left(x^{1} ; x^{2} ; x^{3} ; z\right)$ e a seguinte lagrangiana [10]:

$$
L=\frac{1}{2} \dot{\vec{x}}^{2}-z\left(\vec{x}^{2}-1\right)=\frac{1}{2} \delta_{i j} \dot{x}^{i} \dot{x}^{j}-z\left(\delta_{i j} x^{i} x^{j}-1\right) .
$$

A lagrangiana acima descreve o movimento de uma partícula de massa unitária restrito à superfície de uma esfera centrada na origem de coordenadas e com raio igual à unidade; assim, uma vez que há uma restrição (holônoma ${ }^{21}$ ao movimento livre da partícula, a variável $z$ é introduzida como um multiplicador de Lagrange e prova-se que está diretamente relacionada com a força responsável de manter a restrição ao movimento.

Passemos agora a determinar os momentos canônicos conjugados às variáveis consideradas:

$$
p_{i}=\frac{\partial L}{\partial \dot{x}^{i}}=\delta_{i j} \dot{x}^{j}, \quad p_{z}=\frac{\partial L}{\partial \dot{z}}=0 .
$$

\footnotetext{
${ }^{21}$ Uma restrição é dita holônoma quando pode ser escrita apenas em termos das coordenadas generalizadas; i. e., sem envolver (explicitamente) as velocidades.
}

Assim, as variáveis dinâmicas independentes do espaço de fase são $\xi^{t}=\left(\begin{array}{lll}x^{i} & z & p_{i}\end{array}\right)$. Também, a hamiltoniana correspondente será dada por:

$$
\begin{aligned}
H & =p_{j} \dot{x}^{j}-\frac{1}{2} \delta_{i j} \dot{x}^{i} \dot{x}^{j}+z\left(\delta_{i j} x^{i} x^{j}-1\right) \\
& =\left(p_{j}-\frac{1}{2} \delta_{i j} \delta^{i k} p_{k}\right) \dot{x}^{j}+z\left(\delta_{i j} x^{i} x^{j}-1\right) \\
& =\left(p_{j}-\frac{1}{2} \delta_{j}^{k} p_{k}\right) \delta^{j \ell} p_{\ell}+z\left(\delta_{i j} x^{i} x^{j}-1\right) \\
& =\frac{1}{2} \delta^{i j} p_{i} p_{j}+z\left(\delta_{i j} x^{i} x^{j}-1\right) .
\end{aligned}
$$

Logo, a lagrangiana canônica será simplesmente:

$$
L=p_{i} \dot{x}^{i}-\frac{1}{2} \delta^{i j} p_{i} p_{j}-z\left(\delta_{i j} x^{i} x^{j}-1\right) .
$$

Daqui, identificamos a 1-forma canônica como:

$$
\theta=p_{i} d x^{i}+0 d z+0 d p_{i}
$$

Tomando a derivada exterior obtemos:

$$
\omega=d p_{i} \wedge d x^{i}=\delta_{j}^{i} d p_{i} \wedge d x^{j},
$$

cuja representação matricial será:

$$
\Omega=\begin{aligned}
& d x^{i} \\
& d z \\
& d p_{i}
\end{aligned}\left(\begin{array}{ccc}
d x^{j} & d z & d p_{j} \\
0 & 0 & -\delta_{i}^{j} \\
0 & 0 & 0 \\
\delta_{j}^{i} & 0 & 0
\end{array}\right)
$$

Essa matriz é claramente singular e possui o seguinte autovetor associado ao autovalor nulo:

$$
v^{t}=\left(\begin{array}{lll}
\overrightarrow{0} \in \mathbb{R}^{3} & 1 & \overrightarrow{0} \in \mathbb{R}^{3}
\end{array}\right) .
$$

Com isto, construímos o seguinte vínculo:

$$
\Gamma=\frac{\partial H}{\partial z}=\delta_{i j} x^{i} x^{j}-1=0,
$$

o qual resulta ser precisamente a restrição do movimento à superfície da esfera. Em seguida, determinamos as quantidades em primeira ordem de iteração:

- Hamiltoniana:

$$
H^{(1)}=\left.H\right|_{\Gamma=0}=\frac{1}{2} \delta^{i j} p_{i} p_{j}
$$

- Lagrangiana canônica:

$$
L^{(1)}=p_{i} \dot{x}^{i}+\left(\delta_{i j} x^{i} x^{j}-1\right) \dot{\lambda}-H^{(1)} .
$$

- Coordenadas do espaço de fase $M^{(1)}$ :

Note-se que a variável $z$ não aparece mais nas expressões obtidas para $H^{(1)}$ e $L^{(1)}$, então:

$$
\zeta^{t}=\left(\begin{array}{ll}
x^{i} & p_{i}
\end{array}\right) \quad \Rightarrow \quad \xi^{(1) t}=\left(\begin{array}{lll}
x^{i} & p_{i} & \lambda
\end{array}\right) .
$$


- 1-forma canônica:

$$
\theta^{(1)}=\theta+\left(\delta_{i j} x^{i} x^{j}-1\right) d \lambda .
$$

- Derivada exterior da 1-forma canônica:

$$
\omega^{(1)}=\omega+2 \delta_{i j} x^{j} d x^{i} \wedge d \lambda,
$$

cuja representação matricial será:

$$
\Omega^{(1)}=\left(\begin{array}{cc:c}
0 & -\delta_{i}^{j} & 2 \delta_{i k} x^{k} \\
\delta_{j}^{i} & 0 & 0 \\
\hdashline-2 \delta_{j k} \bar{x}^{-} & 0 & 0
\end{array}\right) .
$$

Novamente, a matriz anterior é singular e possui o seguinte autovetor correspondente ao autovalor nulo:

$$
v^{(1) t}=\left(\begin{array}{lll}
\overrightarrow{0} \in \mathbb{R}^{3} & 2 x^{i} & 1
\end{array}\right),
$$

o que permite construir o vínculo:

$\Gamma^{(1)}=2 \delta_{i j} x^{i} \frac{\partial H^{(1)}}{\partial p_{j}}+\frac{\partial H^{(1)}}{\partial \lambda}=2 \delta_{i j} x^{i} \delta^{j k} p_{k}=2 x^{i} p_{i}=0$

Com isto, já podemos passar à seguinte ordem de iteração:

- Hamiltoniana:

$$
H^{(2)}=\frac{1}{2} \delta^{j k} p_{j} p_{k}
$$

- Lagrangiana canônica:

$$
L^{(2)}=p_{i} \dot{x}^{i}+\left(\delta_{i j} x^{i} x^{j}-1\right) \dot{\lambda}+2 x^{i} p_{i} \dot{\lambda}^{(1)}-H^{(2)} .
$$

- Coordenadas do espaço de fase $M^{(2)}$ :

$$
\xi^{(2) t}=\left(\begin{array}{ll}
\xi^{(1)} & \lambda^{(1)}
\end{array}\right) .
$$

- 1-forma canônica:

$$
\theta^{(2)}=\theta^{(1)}+2 x^{i} p_{i} d \lambda^{(1)} .
$$

- Derivada da 1-forma canônica:

$$
\omega^{(2)}=\omega^{(1)}+2 p_{i} d x^{i} \wedge d \lambda^{(1)}+2 x^{i} d p_{i} \wedge d \lambda^{(1)} .
$$

A representação matricial correspondente é:

$$
\Omega^{(2)}=\left(\begin{array}{cccc}
0 & -\delta_{i}^{j} & 2 \delta_{i k} x^{k} & 2 p_{i} \\
\delta_{j}^{i} & 0 & 0 & 2 x^{i} \\
-2 \delta_{j k} x^{k} & 0 & 0 & 0 \\
-2 p_{j} & -2 x^{j} & 0 & 0
\end{array}\right) .
$$

A matriz $\Omega^{(2)}$ é regular e tem por inversa 22

$$
\left(\begin{array}{cc:cc}
0 & \delta_{j}^{i}-\frac{x^{i} x_{j}}{\vec{x}^{2}} & -\frac{x^{i}}{2 \vec{x}^{2}} & 0 \\
-\delta_{i}^{j}+\frac{x_{i} k x^{j}}{\vec{x}^{2}} & \frac{x_{i} p_{j}-p_{i} x_{j}}{\vec{x}^{2}} & \frac{p_{i}}{2 \vec{x}^{2}} & -\frac{x_{i}}{2 \vec{x}^{2}} \\
\hdashline \frac{x^{j}}{2 \vec{x}^{2}} & -\frac{p_{j}}{2 \vec{x}^{2}} & 0 & -\frac{1}{2 \vec{x}^{2}} \\
0 & \frac{x_{j}}{2 \vec{x}^{2}} & \frac{1}{2 \vec{x}^{2}} & 0
\end{array}\right) .
$$

$\overline{22}$ A divisão com as linhas pontilhadas foi feita entre os graus de liberdade originais e os auxiliares (multiplicadores de Lagrange). Também, temos utilizado a notação $x_{i} \stackrel{!}{=} \delta_{i j} x^{j}$.
Finalmente, obtemos os parênteses de Poisson das variáveis canônicas originais:

$$
\left\{x^{i} ; p_{j}\right\}=\delta_{j}^{i}-\frac{x^{i} x_{j}}{\vec{x}^{2}}, \quad\left\{p_{i} ; p_{j}\right\}=\frac{x_{i} p_{j}-p_{i} x_{j}}{\vec{x}^{2}} .
$$

\subsection{Exemplo 4}

O seguinte exemplo é um pouco diferente dos anteriores, devido à natureza da singularidade do sistema que será analisado. No entanto, o método F-J será implementado da mesma maneira que foi realizado até agora. Considere-se uma partícula movendo-se em um espaço de configuração 3-dimensional em conformidade com a seguinte lagrangiana [16]:

$$
L=\frac{1}{2}\left(\dot{q}_{1}^{2}+\dot{q}_{2}^{2}\right)+\left(q_{1} \dot{q}_{2}-\dot{q}_{1} q_{2}\right) q_{3}+\frac{1}{2}\left(q_{1}^{2}+q_{2}^{2}\right) q_{3}^{2}-V\left(q_{1}^{2}+q_{2}^{2}\right) .
$$

Os momentos canônicos conjugados são:

$$
p_{1}=\dot{q}_{1}-q_{2} q_{3}, \quad p_{2}=\dot{q}_{2}+q_{1} q_{3}, \quad p_{3}=0 .
$$

Na sequência, construímos a hamiltoniana:

$$
H=p_{1} \dot{q}_{1}+p_{2} \dot{q}_{2}-L=\frac{1}{2}\left(p_{1}^{2}+p_{2}^{2}\right)-\left(q_{1} p_{2}-p_{1} q_{2}\right) q_{3}+V .
$$

Note-se que a velocidade $\dot{q}_{3}$ não pode ser obtida em termos das coordenadas e dos momentos; porém, como ela não aparece na lagrangiana, podemos afirmar que a lagrangiana escrita na forma canônica será simplesmente:

$$
L=p_{1} \dot{q}_{1}+p_{2} \dot{q}_{2}-H .
$$

Logo, levando em conta que a variável $p_{3}$ não tem dinâmica, escolhemos as seguintes coordenadas para o espaço de fase:

$$
\xi^{t}=\left(\begin{array}{lllll}
q_{1} & q_{2} & q_{3} & p_{1} & p_{2}
\end{array}\right) .
$$

Com isto, identificamos rapidamente a 1 -forma canônica:

$$
\theta=p_{1} d q_{1}+p_{2} d q_{2}+0 d q_{3}+0 d p_{1}+0 d p_{2} .
$$

Tomando a derivada exterior resulta a 2-forma:

$$
\omega=d p_{1} \wedge d q_{1}+d p_{2} \wedge d q_{2} \equiv\left(\begin{array}{ccccc}
0 & 0 & 0 & -1 & 0 \\
0 & 0 & 0 & 0 & -1 \\
0 & 0 & 0 & 0 & 0 \\
1 & 0 & 0 & 0 & 0 \\
0 & 1 & 0 & 0 & 0
\end{array}\right) .
$$

A matriz anterior é evidentemente singular e tem o seguinte autovetor associado ao autovalor nulo:

$$
v^{t}=\left(\begin{array}{lllll}
0 & 0 & 1 & 0 & 0
\end{array}\right) .
$$

Com isto, construímos o seguinte vínculo:

$$
\Gamma=\frac{\partial H}{\partial q_{3}}=q_{1} p_{2}-p_{1} q_{2}=0 .
$$

A seguir escreveremos as quantidades correspondentes à primeira ordem de iteração: 
- Hamiltoniana:

$$
H^{(1)}=\left.H\right|_{\Gamma=0}=\frac{1}{2}\left(p_{1}^{2}+p_{2}^{2}\right)+V .
$$

- Lagrangiana canônica:

$$
L^{(1)}=p_{1} \dot{q}_{1}+p_{2} \dot{q}_{2}+\left(q_{1} p_{2}-p_{1} q_{2}\right) \dot{\lambda}-H^{(1)} .
$$

- Coordenadas do espaço de fase $M^{(1)}$ :

Note-se que a variável $q_{3}$ não aparece mais e, consequentemente, teremos:

$$
\zeta^{t}=\left(\begin{array}{llll}
q_{1} & q_{2} & p_{1} & p_{2}
\end{array}\right) ; \quad \xi^{(1)}=\left(\begin{array}{c}
\zeta \\
\lambda
\end{array}\right) .
$$

- 1-forma canônica:

$$
\theta^{(1)}=\theta+\left(q_{1} p_{2}-p_{1} q_{2}\right) d \lambda .
$$

- Derivada da 1-forma canônica:

$$
\omega^{(1)}=\omega+\left(q_{1} d p_{2}+p_{2} d q_{1}-p_{1} d q_{2}-q_{2} d p_{1}\right) \wedge d \lambda .
$$

Logo, a matriz associada à 2 -forma $\omega^{(1)}$ é:

$$
\Omega^{(1)}=\left(\begin{array}{ccccc}
0 & 0 & -1 & 0 & p_{2} \\
0 & 0 & 0 & -1 & -p_{1} \\
1 & 0 & 0 & 0 & -q_{2} \\
0 & 1 & 0 & 0 & q_{1} \\
-p_{2} & p_{1} & q_{2} & -q_{1} & 0
\end{array}\right) .
$$

A matriz acima é singular e tem o seguinte autovetor associado ao autovalor nulo:

$$
v^{(1) T}=\left(\begin{array}{lllll}
q_{2} & -q_{1} & p_{2} & -p_{1} & 1
\end{array}\right) .
$$

Com isto, se constrói o seguinte novo vínculo:

$$
\begin{aligned}
\Gamma^{(1)} & =\left(q_{2} \frac{\partial}{\partial q_{1}}-q_{1} \frac{\partial}{\partial q_{2}}+p_{2} \frac{\partial}{\partial p_{1}}\right. \\
& \left.-p_{1} \frac{\partial}{\partial p_{2}}+\frac{\partial}{\partial \lambda}\right) H^{(1)} \\
& =q_{2} \frac{\partial V}{\partial q_{1}}-q_{1} \frac{\partial V}{\partial q_{2}}+p_{2} p_{1}-p_{1} p_{2}+0 \\
& =\left[\left(q_{2} \frac{\partial}{\partial q_{1}}-q_{1} \frac{\partial}{\partial q_{2}}\right)\left(q_{1}^{2}+q_{2}^{2}\right)\right] \frac{\partial V}{\partial\left(q_{1}^{2}+q_{2}^{2}\right)} \\
& =(\underbrace{q_{2}\left(2 q_{1}\right)-q_{1}\left(2 q_{2}\right)}_{0}) \frac{\partial V}{\partial\left(q_{1}^{2}+q_{2}^{2}\right)}=\mathbf{0} .
\end{aligned}
$$

Assim, obtemos a seguinte tautologia (ou identidade):

$$
\Gamma^{(1)}=0=\mathbf{0} .
$$

Então, posto que foi obtido um vínculo nulo, não é possível continuar com o procedimento usual. Estas situações se apresentam com frequência e são próprias dos sistemas que são invariantes por transformações de equivalência ${ }^{23}$ (composição de transformações canônicas infinitesimais que não mudam o estado físico do sistema, no sentido de que o gerador da evolução temporal conduz a um conjunto de pontos do espaço de fase pertencentes a uma classe de equivalência ${ }^{24}$ pois correspondem a um mesmo estado).

Essa degenerescência pode ser removida impondo condições adicionais que restrinjam a dinâmica do sistema de modo que ela se torne unívoca. A proposta do método F-J para enfrentar essa situação é a seguinte:

"No caso de se obter um vínculo nulo na n-ésima ordem de iteração no algoritmo, o vínculo nulo deverá ser substituído por uma condição fixadora $(C F) e$ depois continuar com o procedimento conhecido".

O enunciado anterior pode-se expressar simplesmente como:

$$
\Gamma^{(n)}=0 \rightarrow \Gamma^{\prime(n)}=\mathrm{CF} ; \quad H^{(n+1)}=\left.H^{(n)}\right|_{\Gamma^{\prime(n)}=0} .
$$

Assim, a hamiltoniana da seguinte ordem de iteração, será obtida impondo a condição fixadora à última hamiltoniana construída. É importante fazer uma breve discussão sobre essas condições fixadoras. Em primeiro lugar, essas condições devem ser atingiveis, isto quer dizer que sempre deve ser possível obtê-las por meio de uma transformação de equivalência; essas transformações não devem mudar as equações de movimento, pois devem conduzir aos mesmos estados físicos do sistema.

Não é complicado ver que a lagrangiana (27) permanece invariante sob as seguintes transformações:

$$
\left\{\begin{array}{lllll}
q_{1} & \rightarrow & q_{1}^{\prime} & = & q_{1} \cos (\theta)+q_{2} \operatorname{sen}(\theta) \\
q_{2} & \rightarrow & q_{2}^{\prime}= & -q_{1} \operatorname{sen}(\theta)+q_{2} \cos (\theta) \\
q_{3} & \rightarrow & q_{3}^{\prime} & = & q_{3}
\end{array},\right.
$$

que é, na verdade, uma rotação de um ângulo $\theta$ no plano $q_{1} q_{2}$ mantendo fixa a coordenada $q_{3}$. As rotações no plano são representadas por matrizes $2 \times 2$ especiai: ${ }^{25}$ reais e ortogonai, ${ }^{26}$ com parâmetro $0 \leqslant \theta<2 \pi$. Para o exemplo em consideração, supondo que o ângulo nasce no eixo $q_{1}$, a matriz associada à transformação de coordenadas acima será:

$$
R(\theta)=\left(\begin{array}{cc}
\cos (\theta) & \operatorname{sen}(\theta) \\
-\operatorname{sen}(\theta) & \cos (\theta)
\end{array}\right) \Rightarrow\left(\begin{array}{l}
q_{1}^{\prime} \\
q_{2}^{\prime}
\end{array}\right)=R(\theta)\left(\begin{array}{l}
q_{1} \\
q_{2}
\end{array}\right)
$$

Essas matrizes constituem um grupo (de Lie) denotado por $S O(2)$. Então, diz-se que $S O(2)$ é o grupo de simetria (globa ${ }^{27}$ ) para a lagrangiana (27) quando a coordenada

\footnotetext{
$\overline{23}$ Cujo análogo na teoria de campos é conhecido como transformações de calibre.

24 Uma classe de equivalência é um conjunto de elementos identificados por uma relação (binária) de equivalência; i. e., que é reflexiva, simétrica e transitiva.

25 Uma matriz é dita especial se o determinante dela é 1.

26 Uma matriz $R$ é dita ortogonal se $R^{T} R=1$.

27 Diz-se que a simetria é global quando os parâmetros dos elementos do grupo de simetria são constantes.
} 
$q_{3}$ está fixa. Lembre-se de que as coordenadas $q^{a}$ são, em geral, funções do tempo; então, é interessante ver se a lagrangiana em estudo também torna-se invariante se o parâmetro for também função do tempo; isto é, $\theta=\theta(t)$. Nesse caso ter-se-á:

$$
\begin{aligned}
& \dot{q}_{1}^{\prime}=\dot{q}_{1} \cos (\theta)+\dot{q}_{2} \operatorname{sen}(\theta)+\dot{\theta}\left(-q_{1} \operatorname{sen}(\theta)+q_{2} \cos (\theta)\right), \\
& \dot{q}_{2}^{\prime}=-\dot{q}_{1} \operatorname{sen}(\theta)+\dot{q}_{2} \cos (\theta)-\dot{\theta}\left(q_{1} \cos (\theta)+q_{2} \operatorname{sen}(\theta)\right) .
\end{aligned}
$$

Se a coordenada $q_{3}$ permanece fixa, resulta que a lagrangiana transformada não coincide com a original:

$$
L^{\prime}=L+\frac{1}{2} \dot{\theta}^{2}\left(q_{1}^{2}+q_{2}^{2}\right)-\dot{\theta}\left(q_{1} \dot{q}_{2}-\dot{q}_{1} q_{2}\right)-\dot{\theta}\left(q_{1}^{2}+q_{2}^{2}\right) q_{3} .
$$

Contudo, é possível recuperar a invariância da lagrangiana fazendo uma transformação apropriada à coordenada $q_{3}$. Em primeira instância não é conhecida a forma dessa transformação, pelo qual se considera:

$$
q_{3} \rightarrow q_{3}^{\prime}=q_{3}+\delta q_{3},
$$

Com isto:

$$
\begin{aligned}
L^{\prime}= & L-\left(q_{1} \dot{q}_{2}-\dot{q}_{1} q_{2}\right)\left(\dot{\theta}-\delta q_{3}\right) \\
& +\left(q_{1}^{2}+q_{2}^{2}\right)\left(\frac{1}{2} \dot{\theta}^{2}-\dot{\theta}\left(q_{3}+\delta q_{3}\right)+q_{3} \delta q_{3}+\frac{1}{2}\left(\delta q_{3}\right)^{2}\right) .
\end{aligned}
$$

Logo, observa-se que a invariância da lagrangiana é atingida considerando a seguinte transformação:

$$
q_{3} \rightarrow q_{3}^{\prime}=q_{3}+\dot{\theta} .
$$

Assim, resulta que a lagrangiana 27) é invariante sob as transformações locai: 28

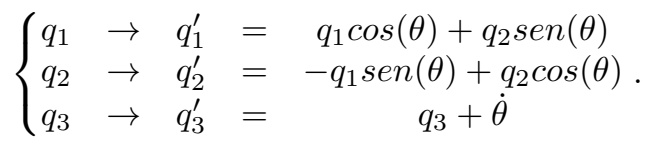

Nesse caso diz-se que a lagrangiana tem simetria local sob o grupo de rotações $S O(2)$. As transformações $\sqrt{28}$ são chamadas de transformações de equivalência.

É possível encontrar uma interpretação da coordenada $q_{3}$ a partir das equações de movimento do sistema:

$$
\begin{aligned}
\text { - } \quad \frac{\delta S}{\delta q_{1}} & =-\ddot{q}_{1}+2 \dot{q}_{2} q_{3}+q_{2} \dot{q}_{3}+q_{1} q_{3}^{2}-\partial_{1} V=0 . \\
\text { - } \quad \frac{\delta S}{\delta q_{2}} & =-\ddot{q}_{2}-2 \dot{q}_{1} q_{3}-q_{1} \dot{q}_{3}+q_{2} q_{3}^{2}-\partial_{2} V=0 . \\
\text { - } \quad \frac{\delta S}{\delta q_{3}} & =-q_{1} \dot{q}_{2}+\dot{q}_{1} q_{2}-\left(q_{1}^{2}+q_{2}^{2}\right) q_{3}=0 .
\end{aligned}
$$

A equação de movimento para $q_{3}$ pode ser escrita como:

$$
\begin{array}{r}
q_{1} \dot{q}_{2}-\dot{q}_{1} q_{2}+\left(q_{1}^{2}+q_{2}^{2}\right) q_{3}=0 \\
q_{1}^{2}\left\{\frac{d}{d t}\left(\frac{q_{2}}{q_{1}}\right)+\left[1+\left(\frac{q_{2}}{q_{1}}\right)^{2}\right] q_{3}\right\}=0
\end{array}
$$

\footnotetext{
$\overline{28}$ Diz-se que as transformações são locais no sentido de que o parâmetro é função da variável $t$.
}

Chamando $z \stackrel{!}{=} \frac{q_{2}}{q_{1}}$, a equação anterior pode ser escrita simplesmente como:

$$
\dot{z}+\left(1+z^{2}\right) q_{3}=0,
$$

que é uma equação de variáveis separáveis; então, podemos fazer o seguinte:

$$
\frac{d z}{z^{2}+1}=-q_{3} d t \quad \rightarrow \quad \int_{z_{0}}^{z} \frac{d z^{\prime}}{z^{\prime 2}+1}=-\int_{0}^{t} q_{3} d t^{\prime} .
$$

Assim:

$$
\tan ^{-1}(z)=\tan ^{-1}\left(z_{0}\right)-\int_{0}^{t} q_{3} d t^{\prime}
$$

Note-se que $\tan ^{-1}(z)=\tan ^{-1}\left(\frac{q_{2}}{q_{1}}\right)$ é o ângulo $\theta$ correspondente à transformação de rotação mencionada acima, então a última equação também pode ser escrita como:

$$
\theta(t)=\theta(0)-\int_{0}^{t} q_{3} d t^{\prime}
$$

Logo, derivando em relação à variável $t$ encontra-se que $q_{3}=-\dot{\theta}$. Assim, a coordenada $q_{3}$ resulta ser o negativo da taxa de câmbio do ângulo de rotação.

O seguinte passo será estabelecer uma condição fixadora para o sistema, a qual é obtida naturalmente a partir da lei de transformação para a coordenada $q_{3}$ :

$$
q_{3}^{\prime}=q_{3}+\dot{\theta}=-\dot{\theta}+\dot{\theta}=0 .
$$

Em consequência:

$$
q_{3}=-\dot{\theta}=0 \Rightarrow \theta=\tan ^{-1}\left(\frac{q_{2}}{q_{1}}\right)=\text { constante }
$$

Desta maneira, obtemos o seguinte vínculd 29 .

$$
\Gamma^{\prime(1)}=\tan ^{-1}\left(\frac{q_{2}}{q_{1}}\right)-c=0 .
$$

Com esse vínculo já é possível passar à seguinte ordem de iteração:

- Hamiltoniana:

$$
H^{(2)}=\frac{1}{2}\left(p_{1}^{2}+p_{2}^{2}\right)+\left.V\left(q_{1}^{2}+q_{2}^{2}\right)\right|_{\tan ^{-1}\left(\frac{q_{2}}{q_{1}}\right)=c} .
$$

- Lagrangiana canônica:

$$
\begin{aligned}
L^{(2)}=p_{1} \dot{q}_{1}+p_{2} \dot{q}_{2}+\left(q_{1} p_{2}-p_{1} q_{2}\right) \dot{\lambda}^{(0)} & \\
& +\Gamma^{(1)} \dot{\lambda}^{(1)}-H^{(2)} .
\end{aligned}
$$

$\overline{29}$ Poderíamos ter tomado como vínculo a condição $q_{3}=0$; porém, não teria resultado conveniente devido ao fato de que a variável $q_{3}$ não formava mais parte das coordenadas do espaço de fase $M^{(1)}$. 
- Coordenadas do espaço de fase $M^{(2)}$ :

$$
\xi^{(2)}=\left(\begin{array}{l}
\xi^{(1)} \\
\lambda^{(1)}
\end{array}\right) .
$$

- 1-forma canônica:

$$
\theta^{(2)}=\theta^{(1)}+\left[\tan ^{-1}\left(\frac{q_{2}}{q_{1}}\right)-c\right] d \lambda^{(1)} .
$$

- Derivada da 1-forma canônica:

$$
\omega^{(2)}=\omega^{(1)}+\left(\frac{q_{1} d q_{2}-q_{2} d q_{1}}{q_{1}^{2}+q_{2}^{2}}\right) \wedge d \lambda^{(1)} .
$$

Logo, a representação matricial $\Omega^{(2)}$ será:

$$
\left(\begin{array}{cccccc}
0 & 0 & -1 & 0 & p_{2} & -\frac{q_{2}}{q_{1}^{2}+q_{2}^{2}} \\
0 & 0 & 0 & -1 & -p_{1} & \frac{q_{1}}{q_{1}^{2}+q_{2}^{2}} \\
1 & 0 & 0 & 0 & -q_{2} & 0 \\
0 & 1 & 0 & 0 & q_{1} & 0 \\
-p_{2} & p_{1} & q_{2} & -q_{1} & 0 & 0 \\
\frac{q_{2}}{q_{1}^{2}+q_{2}^{2}} & -\frac{q_{1}}{q_{1}^{2}+q_{2}^{2}} & 0 & 0 & 0 & 0
\end{array}\right) .
$$

A matriz anterior é regular e tem por inversa:

$\left(\begin{array}{cccc:cc}0 & 0 & \frac{q_{1}^{2}}{q_{1}^{2}+q_{2}^{2}} & \frac{q_{1} q_{2}}{q_{1}^{2}+q_{2}^{2}} & 0 & q_{2} \\ 0 & 0 & \frac{q_{1} q_{2}}{q_{1}^{2}+q_{2}^{2}} & \frac{q_{2}^{2}}{q_{1}^{2}+q_{2}^{2}} & 0 & -q_{1} \\ \frac{-q_{1}^{2}}{q_{1}^{2}+q_{2}^{2}} & \frac{-q_{1} q_{2}}{q_{1}^{2}+q_{2}^{2}} & 0 & \frac{p_{2} q_{1}-q_{2} p_{1}}{q_{1}^{2}+q_{2}^{2}} & \frac{q_{2}}{q_{1}^{2}+q_{2}^{2}} & p_{2} \\ \hdashline \frac{-q_{1} q_{2}}{q_{1}^{2}+q_{2}^{2}} & \frac{-q_{2}^{2}}{q_{1}^{2}+q_{2}^{2}} & \frac{q_{2} p_{1}-p_{2} q_{1}}{q_{1}^{2}+q_{2}^{2}} & 0 & \frac{-q_{1}}{q_{1}^{2}+q_{2}^{2}} & -p_{1} \\ 0 & 0 & \frac{-q_{2}}{q_{1}^{2}+q_{2}^{2}} & \frac{q_{1}}{q_{1}^{2}+q_{2}^{2}} & 0 & 1 \\ -q_{2} & q_{1} & -p_{2} & p_{1} & -1 & 0\end{array}\right)$

Finalmente, lembrando do vínculo $\Gamma^{(0)}$, os parênteses de Poisson não-nulos resultam:

$$
\begin{gathered}
\left\{q_{1} ; p_{1}\right\}=\frac{q_{1}^{2}}{q_{1}^{2}+q_{2}^{2}}, \quad\left\{q_{1} ; p_{2}\right\}=\frac{q_{1} q_{2}}{q_{1}^{2}+q_{2}^{2}}=\left\{q_{2} ; p_{1}\right\} \\
\left\{q_{2} ; p_{2}\right\}=\frac{q_{2}^{2}}{q_{1}^{2}+q_{2}^{2}} .
\end{gathered}
$$

\section{Comentários finais}

A formulação da Mecânica à la Faddeev-Jackiw é perfeitamente compatível com a abordagem hamiltoniana, pois elas compartem a mesma estrutura canônica, sendo uma clara evidência a possibilidade de se obter os parênteses de Poisson. Contudo, a mecânica hamiltoniana tal como a conhecemos fica restrita ao caso de sistemas nãosingulares, enquanto que a formulaçã ${ }^{30}$ F-J oferece uma alternativa para o tratamento de sistemas singulares, inclusive para aqueles cuja singularidade provém das simetrias internas; nesse sentido, a abordagem F-J é mais geral.

\footnotetext{
$\overline{30}$ Com isto estamos nos referindo ao método F-J para sistemas singulares (com o aporte de Barcelos-Neto e Wotzasek).
}

Um aspecto relevante da formulação F-J é a liberdade que temos de escolher as coordenadas mais apropriadas para o espaço de fase apenas escrevendo a lagrangiana na forma canônica, pois as expressões para os momentos canônicos nos dizem quando existe alguma dependência entre alguns dos momentos e as coordenadas, o que é refletido na não-independência linear entre as formas $d q^{a}$ e $d p_{a}$, o que, ademais, implica que com essas coordenadas não poderia se construir uma base apropriada para o espaço de 1-formas no espaço de fase. Por outro lado, a compatibilidade dos vínculos com as equações de movimento é devido ao fato de que estas foram utilizadas na obtenção deles.

Não podemos deixar de mencionar a enorme relevância que tem a 1-forma canônica na abordagem F-J, ela é uma peça chave para a construção da formulação F-J, pois a sua derivada exterior fornece o objeto que vai determinar o caráter singular ou regular do sistema, além de conter a estrutura canônica da teoria na iteração que haja sido construída. Como já foi apresentado, o objetivo final é poder obter uma forma simplética no espaço de fase, pois é com a sua inversa que podemos determinar a estrutura de Poisson desejada que nos permitirá, em princípio, proceder com a quantização canônica.

Nosso último exemplo pode servir de inspiração para se fazer um estudo mais detalhado das teorias com simetrias internas na formulação F-J. Isso será apresentado em um trabalho posterior quando a abordagem seja implementada em sistemas contínuos, especificamente teorias de campos clássicos, pois a maioria delas apresentam as chamadas simetrias de calibre, cujo estudo é de enorme importância tanto em nível clássico quanto quântico. É de salientar, também, que no estudo de teorias de campos, existem campos que descrevem um tipo especial de partículas chamadas de férmions que são caracterizados pelo fato de apresentar spin semi-inteiro, como, por exemplo, o elétron e a sua antipartícula: o pósitron; matematicamente resulta mais apropriado modelar esses campos com um tipo especial de variáveis, conhecidas como números de Grassmann, que têm a propriedade de anticomutar, tal como os campos femiônicos. Assim, existem teorias com graus de liberdade discretos que envolvem esse tipo de números, o que concretamente é chamado de mecânica pseudoclássica, ou simplesmente pseudomecânica e é claro que a abordagem F-J pode ser desenvolvida, mas tomando os cuidados necessários pelo fato de não trabalhar no corpo dos números reais.

\section{Agradecimentos}

L. G. Caro agradece à CAPES pelo suporte integral, B. M. Pimentel agradece ao $\mathrm{CNPq}$ pelo suporte parcial, G. E. R. Zambrano agradece a "Vicerrectoría de Investigaciones e Interacción Social de la Universidad de Nariño", Projects No. 1928 and No. 2172. Os autores agradecem ao (à) revisor (a) pelos comentários e sugestões que permitiram melhorar nosso artigo. 


\section{Referências}

[1] P.A.M. Dirac, Proceedings of the Royal Society of London Series A 109, 642 (1925).

[2] P.G. Bergmann, Physical Review 75, 680 (1949).

[3] P.G. Bergmann e J.H. Brunings, Reviews of Modern Physics 21, 480 (1949).

[4] P.G. Bergmann, R. Penfield, R. Schiller e H. Zatzkis, Physical Review 80, 81 (1950).

[5] J.L. Anderson e P.G. Bergmann, Physical Review 83, 1018 (1951).

[6] P.A.M. Dirac, Canadian Journal of Mathematics 2, 129 (1950).

[7] P.A. Dirac, Canadian Journal of Mathematics 3, 1 (1951).

[8] L. Faddeev e R. Jackiw, Physical Review Letters 60, 1692 (1988).

[9] J. Barcelos-Neto e C. Wotzasek, International Journal of Modern Physics A 7, 4981 (1992).

[10] J. Barcelos-Neto e C. Wotzasek, Modern Physics Letters A 7, 1737 (1992).

[11] K. Sundermeyer, Constrained dynamics with applications to Yang- Mills theory, general relativity, classical spin, dual string model (Springer-Verlag, Berlin, 1982).

[12] R. Abraham e J.E. Marsden, Foundations of Mechanics (Addison-Wesley Publishing Company, Redwood City, 1987).

[13] L.E. Elsgolts, Differential equations and the calculus of variations (Mir Publishers, Moscow, 1977).

[14] G.V. Dunne, R. Jackiw e C.A. Trugenberger, Physical Review D 41, 661 (1990).

[15] W.M. Seiler, Journal of Physics A: Mathematical and General 28, 7315.(1995).

[16] N.H. Christ e T.D. Lee, Physical Review D 22, 939 (1980). 